\title{
Huntingtin Bodies Sequester Vesicle-Associated Proteins by a Polyproline-Dependent Interaction
}

\author{
Zheng-Hong Qin, ${ }^{1}$ Yumei Wang, ${ }^{1}$ Ellen Sapp, ${ }^{1}$ Benjamin Cuiffo, ${ }^{1}$ Erich Wanker, ${ }^{3}$ Michael R. Hayden, ${ }^{4}$ \\ Kimberly B. Kegel, ${ }^{1}$ Neil Aronin, ${ }^{2}$ and Marian DiFiglia ${ }^{1}$ \\ ${ }^{1}$ Laboratory of Cellular Neurobiology, Massachusetts General Hospital and Harvard Medical School, Charlestown, Massachusetts 02129, ${ }^{2}$ Departments of \\ Medicine and Cell Biology, University of Massachusetts Medical Center, Worcester, Massachusetts 01655, ${ }^{3}$ Max-Planck-Institut for Molecular Genetics, \\ D-14195 Berlin, Germany, and ${ }^{4}$ Molecular Medicine and Therapeutics, University of British Columbia, Vancouver, British Columbia V5Z 4H4, Canada
}

Polyglutamine expansion in the $\mathrm{N}$ terminus of huntingtin (htt) causes selective neuronal dysfunction and cell death by unknown mechanisms. Truncated htt expressed in vitro produced htt immunoreactive cytoplasmic bodies (htt bodies). The fibrillar core of the mutant $\mathrm{htt}$ body resisted protease treatment and contained cathepsin D, ubiquitin, and heat shock protein (HSP) 40 . The shell of the htt body was composed of globules $14-34 \mathrm{~nm}$ in diameter and was protease sensitive. HSP70, proteasome, dynamin, and the htt binding partners htt interacting protein 1 (HIP1), SH3-containing Grb2-like protein (SH3GL3), and 14.7K-interacting protein were reduced in their normal location and redistributed to the shell. Removal of a series of prolines adjacent to the polyglutamine region in htt blocked formation of the shell of the htt body and redistribution of dynamin, HIP1, SH3GL3, and proteasome to it. Internalization of transferrin was impaired in cells that formed htt bodies. In cortical neurons of Huntington's disease patients with early stage pathology, dynamin immunoreactivity accumulated in cytoplasmic bodies. Results suggest that accumulation of a nonfibrillar form of mutant htt in the cytoplasm contributes to neuronal dysfunction by sequestering proteins involved in vesicle trafficking.

Key words: Huntington's disease; huntingtin; autophagy; vesicle trafficking; dynamin; protein aggregates; protein interactions

\section{Introduction}

Huntington's disease (HD) is an autosomal dominant disease resulting in the expansion of a polyglutamine tract in huntingtin (htt). Neuropathology of HD is characterized by selective neuronal loss in the striatum and cortex. N-terminal fragments of mutant htt form insoluble aggregates in the nucleus and cytoplasm of affected neurons in the HD brain (DiFiglia et al., 1997). The aggregates formed in HD brain have amyloid-like fibrils. $\mathrm{N}$-terminal mutant htt encoded by exon 1 (aa 1-89 in normal htt) also forms amyloid-like fibrils in vitro (Scherzinger et al., 1997; Hazeki et al., 2002; Poirer et al., 2002). The role of mutant htt aggregates in cell death is controversial, because the presence of aggregates does not consistently correlate with cell death (Saudou et al., 1998; Sisodia 1998; Kim et al., 1999; Kuemmerle et al., 1999). A soluble, nonaggregated, mutant htt might contribute to neurodegeneration by engaging in abnormal protein interactions (Bao et al., 1996; Sittler et al., 1998; Passani et al., 2000; Gervais et al., 2002). Oligomers of mutant htt expressed from exon 1 form spheroids and protofibrils as intermediates (prefibrillar forms)

Received May 9, 2003; revised 0ct. 1, 2003; accepted 0ct. 20, 2003.

This work was supported by National Institutes of Health Grants NS16367 (M.D.), NS13579 (M.D.), and NS38194 (N.A.) and by a grant from the Huntington's Disease Society of America (M.D.). We thank Dr. Alfred Heller from the University of Chicago (Chicago, IL) for providing us with $\times 57$ cells, Dr. Xiao-Jiang Li from Emory University (Atlanta, GA), and Dr. Stephen Hersch from Massachusetts General Hospital (Charlestown, MA) for providing us with EM48 antibody.

Correspondence should be addressed to Dr. Zheng-Hong Qin, Laboratory of Cellular Neurobiology, Massachusetts General Hospital, 114 16th Street, Room 2125, Charlestown, MA 02129-4404. E-mail: zhqin5@hotmail.com. DOI:10.1523/JNEUROSCI.1409-03.2004

Copyright $\odot 2004$ Society for Neuroscience $\quad$ 0270-6474/04/240269-13\$15.00/0 during fibril assembly (Poirier et al., 2002). Although the toxic effects of either prefibrillar or soluble, nonfibrillar forms of mutant htt have not yet been determined in cell models of HD, it has been reported that stable, soluble oligomers of $\beta$-amyloid 1-42 have neurotoxic effects (Walsh et al., 2002).

We showed that truncated wild-type (wt) or mutant htt 1-969 aa expressed in clonal striatal cells assembled into cytoplasmic structures that contained the lysosomal enzyme cathepsin D (Kegel et al., 2000). At the EM level, some of the htt was bound to early and late autophagososomes, which are prelysosomal structures involved in the removal of cytoplasmic proteins. Immunofluorescence microscopy suggested that htt-labeled autophagosomes fuse with each other to form larger and more condensed bodies. In this study, we refer to the coalesced, htt-labeled autophagosomes as htt bodies. It is unclear whether htt bodies form in neurons of $\mathrm{HD}$ patients to the same extent that they do in vitro and have a pathological role. Marked accumulations of htt in the cytoplasm of HD neurons are associated with multivesicular bodies, a morphology characteristic of autophagosomes (Sapp et al., 1997). Because they accumulate htt, htt bodies might be sites to identify different forms of htt and to study the interaction of htt with other cytoplasmic proteins. The polyproline region that lies adjacent and distal to the polyglutamine tract in htt is a sequence potentially capable of different interactions with proteins that have Src homology 3 (SH3) and WW domains (Liu et al., 1997; Faber et al., 1998; Sittler et al., 1998; Passani et al., 2000). The proline stretch may also confer structural stability to the $\mathrm{N}$-terminal region of htt. Therefore, we speculated that a deletion 
of the polyproline region in htt might affect htt structure, the formation of htt bodies, and htt interaction with binding partners.

Our results showed that distinct forms of htt are partitioned into a core and shell of the htt body. Mutant htt in the core was fibrillar and protease resistant. The htt in the shell formed oligomeric 14-34 nm globules and reduced and sequestered cytoplasmic proteins involved in vesicle trafficking, removing them from their normal locations in the cytoplasm. Polyglutamine expansion increased formation of htt bodies. Polyproline deletion reduced formation of htt bodies and prevented the redistribution of cytoplasmic proteins to the shell. The htt bodies impaired the transport of transferrin. Cortical neurons of grade 1/presymptomatic HD patients show altered distribution of dynamin, a sign that early dysfunction in endocytosis may occur. Our findings suggest that soluble oligomers of mutant htt that assemble at the periphery of htt bodies may cause cell dysfunction in HD, by sequestering proteins involved in vesicle trafficking through a polyproline-dependent mechanism.

\section{Materials and Methods}

Plasmid construction. The construction of expression plasmids of fulllength htt and truncated htt has been described previously (Kim et al., 1999). A Flag tag was fused to the $\mathrm{N}$ terminus of htt with $18 \mathrm{Q}$ for wt htt and 46Q or 100Q for mutant htt. In this study, these constructs are denoted as htt with Flag tag (FH) 3144-18, FH3144-100 (for full-length htt), and FH969-18, FH969-46 and FH969-100 for truncated htt. The numbered residues in htt are based on the $23 \mathrm{Q}$ htt sequence. In addition, constructs were made encoding Flag fused to the $\mathrm{N}$ terminus of htt $1-171,1-287$, or 1-400 with different glutamine repeats (FH171-18, FH171-100, FH287-18, FH287-100, FH400-18, and FH400-100). For deletion of the region encoding prolines, htt cDNA was isolated by $B a m H I(314) \rightarrow X h o I ~(823)$ digestion and treated with AciI, which cuts at bases encoding prolines. Two DNA bands were isolated from a gel: BamHI-AciI (bp 314-436) and AciI-XhoI (bp 571-823). A smaller band (bp 436-571) was lost during isolation. BamHI-AciI and AciI-XhoI were ligated together, and BamHI $\rightarrow$ XhoI was then cloned into BamHI NsiI of pcDNA-FLAG vectors containing 316-3221 bp of the htt gene with 18 or 46 CAG repeats. DNA sequencing confirmed that these procedures yielded a deletion of nucleotides that encode residues between aa 41-89 that include 30 prolines. The modified expression vectors were designated FH969-18 (proline-) and FH969-46 (proline-). A similar modification of truncated htt cDNA bp 316-3221 with 100 CAG repeats was unsuccessful and could not be used in this study.

Cell culture and DNA transfections. Three cells lines were used in this study. Mouse clonal striatal cells (x57) were developed by fusion of mouse day 17 embryonic striatum and mouse neuroblastoma cells (Wainwright et al., 1995). COS1 cells and MCF-7 cells were obtained from American Type Culture Collection (Manassas, VA). COS1 cells are SV40-transformed kidney fibroblasts from African green monkey. MCF-7 cells are epithelial-type cells derived from human mammary gland carcinoma. Mouse clonal striatal x 57 cells (a generous gift from Dr. Alfred Heller, University of Chicago, Chicago, IL) and MCF-7 cells were cultured in $60 \mathrm{~mm}$ dishes in DMEM with 10\% FBS, 4.5\% glucose, and 50 $\mathrm{U} / \mathrm{ml}$ penicillin $\mathrm{G}$-streptomycin (Kim et al., 1999). MCF-7 and COS1 cells were cultured in $60 \mathrm{~mm}$ dishes in DMEM with $10 \% \mathrm{FBS}$ and $50 \mathrm{u} / \mathrm{ml}$ penicillin G-streptomycin. At $80 \%$ confluence, cells were incubated in 2 $\mathrm{ml}$ of DMEM containing 5-10 $\mu \mathrm{g}$ of htt cDNA and $60 \mu \mathrm{l}$ of Superfect (Qiagen, Valencia, CA). After $3 \mathrm{hr}$ of incubation, cells were given $6 \mathrm{ml}$ of fresh DMEM, and culturing was continued to the desired time or cells were split into 24 -well plates containing poly-L-lysine-coated cover slides for use in immunohistochemistry.

Immunohistochemistry in cultured cells and brain tissue. $\mathrm{x} 57, \mathrm{COS} 1$, or MCF-7 cells were cultured on poly-L-lysine-coated microslips for 6-24 $\mathrm{hr}$ after transfection of different cDNAs encoding Flag-htt fusion proteins as described above. Cells were fixed with $4 \%$ paraformaldehyde in PBS and processed for immunohistochemistry as described previously
(Qin et al., 2001). The formation of htt bodies or htt aggregates was detected with antibody against Flag (monoclonal antibody M5; Sigma, St. Louis, MO) and antibodies that recognize different htt epitopes including monoclonal antibody 2166 (aa 414-503 in htt; Chemicon, Temecula, CA), rabbit polyclonal antibodies Abl (aa 1-17 in htt), Ab585 (aa 585-725 in htt), and EM48 [aa 1-257 of htt without the polyglutamine and polyproline domains; a generous gift from Drs. Xiao-Jiang Li (Emory University School of Medicine, Atlanta, GA) and Stephen Hersch (Massachusetts General Hospital and Harvard Medical School, Charlestown, MA)]. Immunostained cells were examined with a confocal microscope (Radiance 2001; Bio-Rad, Hercules, CA) using a $100 \times$ oil immersion lens. Confocal images were captured with Laser Sharp 2000 software and merged in Adobe Photoshop (see more details below).

For double-labeling studies, anti-htt antibodies 2166 and EM48 were used. Anti-Flag antibody M5 was combined with rabbit polyclonal antibodies against htt interacting protein 1 (HIP1), SH3-containing Grb2like protein (SH3GL3), heat shock protein (HSP) 40 (Santa Cruz Biotechnology, Santa Cruz, CA), HSP60 (Stress Genes, Victoria, British Columbia, Canada), HSP70 (Santa Cruz Biotechnology), cathepsin D (Calbiochem, San Diego, CA), active caspase-3 (Cell Signaling Technology, Beverly, MA), and proteasome (a generous gift from Dr Josè G. Castaño, Universidad Autónoma de Madrid, Madrid, Spain). Anti-htt polyclonal antibody Ab1 was combined with mouse monoclonal antibodies against 14.7K-interacting protein (FIP2; Transduction Laboratories, Lexington, KY), dynamin (PharMingen, San Diego, CA), ubiquitin (Santa Cruz Biotechnology), and dynamin-like protein 1 (DLP1; PharMingen). The specificity of antibodies has been validated before or tested in the present study by omission of primary or secondary antibodies. Secondary antisera included Bodipy fluorophore conjugated goat antimouse (Molecular Probes, Eugene, OR) and Cy3-conjugated donkey anti-mouse (Jackson Immunoresearch, West Grove, PA). The analysis of colocalization of cytoplasmic proteins in htt bodies was performed using a Krypton-Argon laser that scanned immunostained cells sequentially with single emission filters installed to detect Bodipy FL (excitation/ emission maxima, 503/512) and Cy3 (excitation/emission maxima, 550/ 565). Single images were obtained after excitation at $488 \mathrm{~nm}$ to detect Bodipy FL or at $568 \mathrm{~nm}$ to detect Cy3, then merged using Adobe Photoshop. The sequential imaging procedure ensured that two images were taken at the same focal plane and avoided cross-excitation of the immunofluorescent labeling.

Immunoperoxidase labeling to detect dynamin or htt immunoreactivity was performed on $7 \mu \mathrm{m}$ thick slide-mounted frozen sections of postmortem frontal cortex from human control brain $(n=3)$ and adult onset HD patient brain $(n=3)$. The method for immunoperoxidase labeling in human brain sections has been described previously (Sapp et al., 1995). The sections were pretreated with $10 \%$ methanol and $1 \% \mathrm{H}_{2} \mathrm{O}_{2}$ for 15 $\mathrm{min}$ at room temperature before the immunostaining procedure. Polyclonal antibody EM48 (dilution, 1:1000) was used to detect htt. The mean age of controls and HD patients was 64 and 55 years, respectively. The postmortem interval was $17 \mathrm{hr}$ for controls and $25 \mathrm{hr}$ for HD cases. The control (C10, C12, and C18) and HD (A4, A13, A20) brains have been used in our previous published studies of htt localization (DiFiglia et al., 1997; Sapp et al., 1999). Two of the HD brains (A4 and A13) had grade 1 neuropathology, which signifies minimal loss of striatal neurons (Vonsattel et al., 1985). Both cases were verified to have an expanded CAG repeat in the HD gene. A4 was an asymptomatic patient. The other HD brain had grade 3 striatal pathology, which indicates significant striatal cell loss. Pyramidal cortical neurons are relatively preserved in grade $1 \mathrm{HD}$ brain and depleted in grade $3 \mathrm{HD}$ brain (Vonsattel and DiFiglia, 1998).

In situ proteinase K degradation of htt bodies. MCF-7 cells were cultured on poly-L-lysine-coated microslips and transfected with FH969-100. Cells were fixed in $4 \%$ paraformaldehyde in PBS $3 \mathrm{~d}$ after htt expression. Cells were washed in PBS and permeabilized with $0.1 \%$ Triton X-100 in PBS and then incubated with $0.25-27 \mu \mathrm{g} / \mathrm{ml}$ proteinase $\mathrm{K}$ in PBS for 10-40 min at room temperature. Cells were then washed in PBS and processed for double immunostaining using antisera 2166 and EM48 or dynamin and EM48. Degradation of htt bodies by proteinase K was analyzed with a laser confocal microscope as described above. 
A

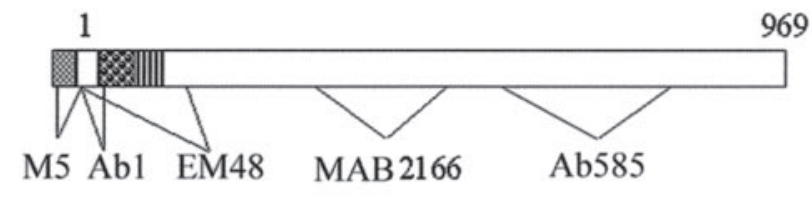

Htt aa

$$
1-17 \quad 1-257 \quad 414-503
$$

$585-725$

\section{: Flag Tag PolyQ18, 46 or 100}

B
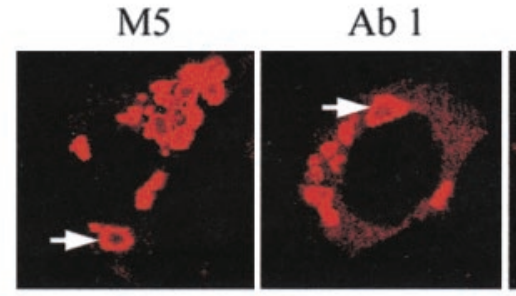
EM 48
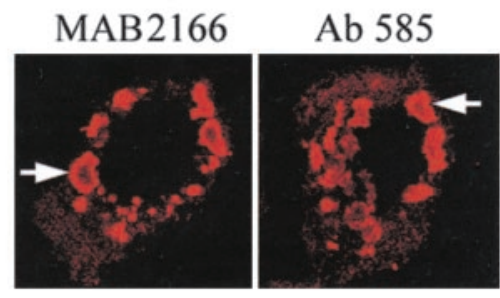

\section{C}
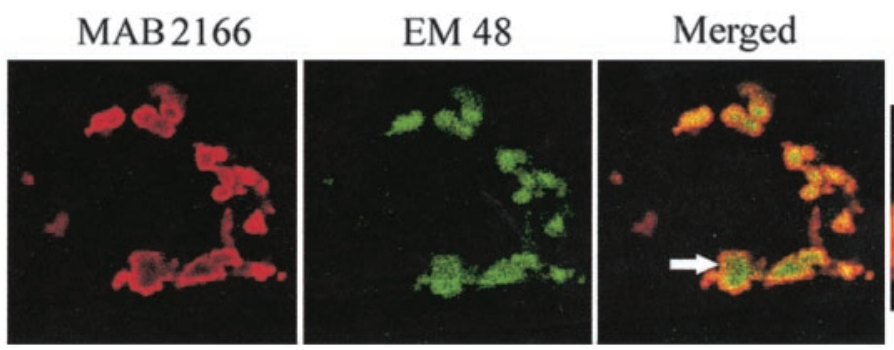

Figure 1. Organization of htt bodies. A, Diagram shows Flag-htt with aa 1-969 of htt. The location of the Flag tag and the polyglutamine and polyproline regions is indicated. Also identified are the locations of epitopes and corresponding antibodies used to study htt bodies. B, Confocal immunofluorescence microscopy of htt bodies in MCF-7 cells that were treated with antisera against Flag (M5) or htt (Ab1, EM 48, mAb2166, and Ab585). The htt bodies are identified by rings of intense labeling (arrows) with all antisera, except EM48, which produces solid patches of htt immunoreactivity. C, Double immunostaining with antisera 2166 and EM48 reveals the partitioning of different htt epitopes into the shell (labeled by 2166) and the core (labeled by EM48). Cells were transfected with FH969-100. The htt body at the arrow in the merged image is enlarged at right. The enlarged htt body shows three zones of immunoreactivity: the core labeled by EM48 (green); an outer region labeled by antibody 2166 (red, arrowhead); and a transitional region labeled by EM48 and 2166 (yellow, arrow).

Quantification of htt bodies. X57 or MCF-7 cells were cultured on poly-L-lysine-coated microslips and transfected with FH969-18 or FH969-100, FH969-18 (proline-) or FH969-46, or FH969-46 (proline-). Cells were fixed and processed for immunohistochemistry using M5 and 2166. The slides were processed by an observer blind to the treatment conditions. One thousand Flag-positive cells on each microslip were examined for the expression of diffuse htt and htt bodies using a fluorescence microscope with $60 \times$ objective and an ocular grid (Eclipse TE 300; Nikon, Tokyo, Japan). The data were statistically analyzed with Student's $t$ test or ANOVA and presented in bar figures as the number of cells with htt bodies per 1000 cells expressing exogenous htt. To quantify the number of cells colocalizing a cytoplasmic protein in htt bodies, 100 cells with htt bodies/slide were analyzed with a confocal microscope with $100 \times$ oil immersion objective.

EM examination. MCF-7 cells were cultured onto poly-L-lysine-coated microslips and transfected with FH969-18 or FH969-100, or FH287-18 or FH287-100. Cells were fixed in 4\% paraformaldehyde in PBS $3 \mathrm{~d}$ after

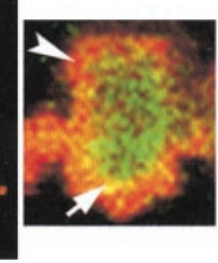

transfection. Cells were incubated with antibody M5 and horseradish peroxidaseconjugated anti-mouse IgG antibody. The htt bodies were visualized with DAB. Cells were postfixed in $2.5 \%$ glutaraldehyde, incubated in $1 \%$ osmium tetroxide and $1 \%$ uranyl acetate, dehydrated in increasing grades of alcohol, and embedded in an ethanol-soluble resin (LX112, LADD). Embedded cells were sectioned (Ultracut E; Reichert-Jung) and examined with a JEOL 100CX electron microscope.

Transferrin uptake assay. MCF-7 cells were cultured onto poly-L-lysine-coated microslips and transfected with FH969-18 or FH969-100. After $24 \mathrm{hr}$ of htt expression, culture medium was removed, and cells were incubated in DMEM containing $2.0 \mathrm{~mm}$ HEPES and $0.2 \%$ BSA for $45 \mathrm{~min}$ at $37^{\circ} \mathrm{C}$ with $5.0 \% \mathrm{CO}_{2}$ to remove surface-bound transferrin. The cells were washed twice with ice-cooled buffer containing $100 \mathrm{~mm}$ potassium acetate, $85 \mathrm{~mm}$ sucrose, 1 mM magnesium acetate, and $20 \mathrm{~mm}$ HEPES$\mathrm{NaOH}$ (KSHM; pH 7.4). Microslips were incubated in $0.4 \mathrm{ml}$ of KSHM with $0.2 \%$ BSA and 6.0 $\mu \mathrm{g} / \mathrm{ml}$ human transferrin conjugated to tetramethylrhodamine at $37^{\circ} \mathrm{C}$ with $5 \% \mathrm{CO}_{2}$ for $5-45$ min. The cells were washed with cold KSHM buffer three times, fixed in $4 \%$ paraformaldehyde, and processed for immunohistochemical localization of Flag using M5 antibody and Bodipy FL-conjugated anti-mouse secondary antibody. Uptake of transferrin in nontransfected cells, in cells with diffuse htt expression and in cells with htt bodies was quantified with a fluorescence microscope using a $60 \times$ objective by an observer (E.S.) who had no knowledge of the treatment conditions. The uptake of transferrin in htt-expressing cells was rated as the same, significantly reduced (by at least $50 \%$ ), or absent compared with nontransfected cells that were adjacent to the htt-expressing cells within the same microscopic field. The data were statistically analyzed with Student's $t$ test. For illustration, confocal images were obtained as described above, and images were merged with Adobe Photoshop.

\section{Results}

\section{Different htt epitopes partition in}

htt bodies

We previously found that transient expression of Flag-htt 1-969 aa (FH969 with 18 glutamines for wild type or 46 or 100 glutamines for mutant type) in clonal striatal cells (x57 cells) produced htt immunofluorescent structures in the cytoplasm that accumulate the autophagosomal/lysosomal enzyme cathepsin D (Kim et al., 1999; Kegel et al., 2000). By electron microscopy, the htt exressed in the cytoplasm was associated with autophagosome-like bodies (Kegel et al., 2000). The formation of htt-labeled bodies coincided with the generation of N-terminal htt (N-htt) fragments. Cells with htt-labeled autophagic bodies were significantly smaller than cells without these structures. Polyglutamine expansion increased the remnants of dying cells with htt-labeled autophagic bodies (Kim et al., 1999). These findings suggested that the accumulation of mutant htt-labeled autophagic bodies contributed to cell death. A time course study of x 57 cells showed that httlabeled autophagic structures in the cytoplasm are first detected 

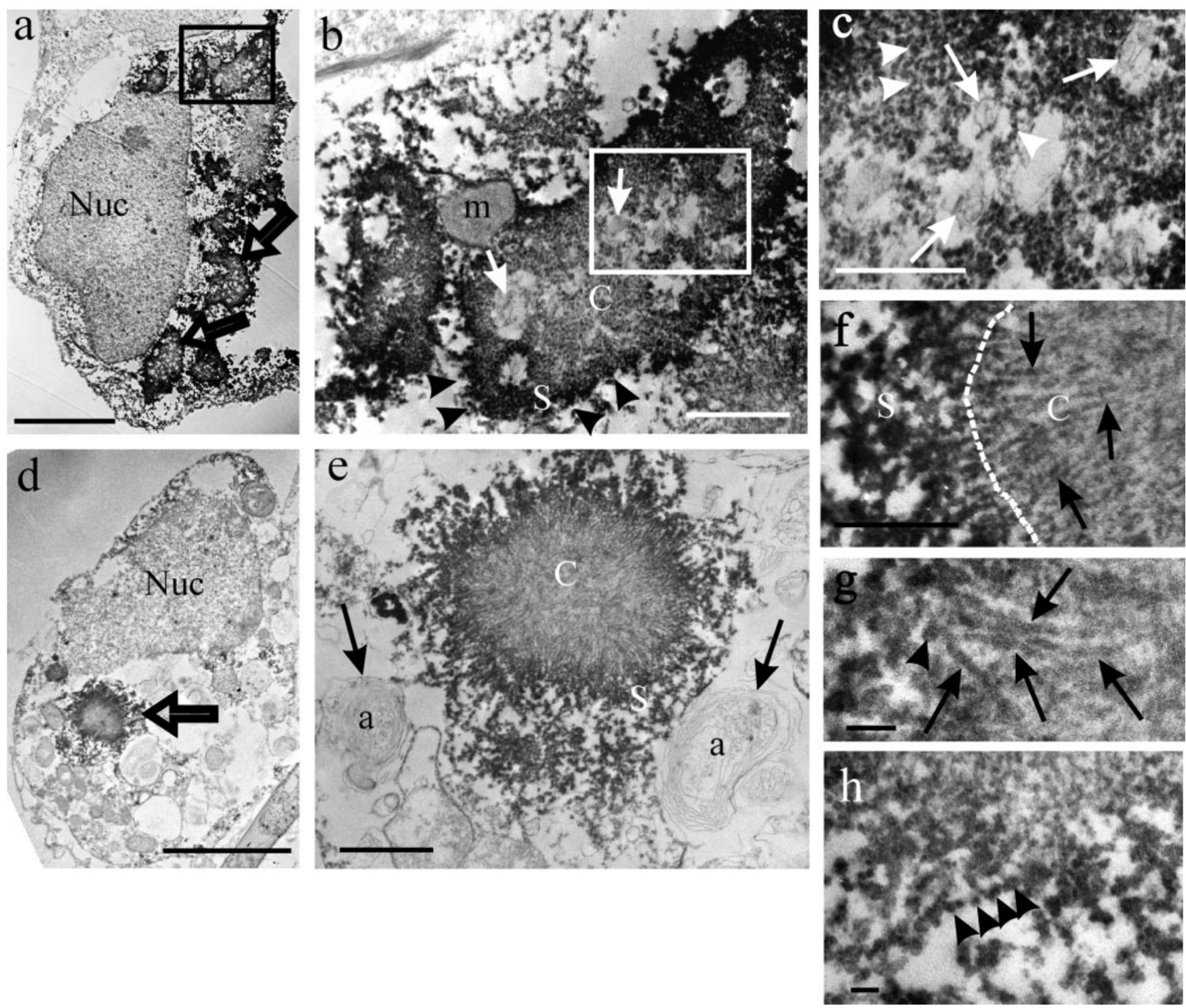

Figure 2. Ultrastructure of htt bodies. MCF-7 cells were transiently transfected with FH969-100Q and immunostained for FLAG using the immunoperoxidase method. $a$, Electron micrograph of a cell with many htt bodies (arrows). Note the intense immunoperoxidase labeling at the rim but weak labeling in the core. The cytoplasm surrounding htt bodies is devoid of organelles. The nucleus (Nuc) is intact and has some small focal patches of immunoreactivity. The dense structure toward the top is the nucleolus. $b$, Higher magnification of boxed region at top right in $a$ shows two htt bodies, a small one on the left and a larger one on the right. Note the intense immunoperoxidase reaction product (arrowheads) along the shell (S) of the large htt body. There is a mitochondrion ( $\mathrm{m}$ ) partly engulfed by the shell. Multivesicular bodies are in the core (C; arrows). The boxed region is shown in c.c, The core of the htt body contains tortuous fibrils (arrowheads) and multivesicular bodies (arrows). $d$, A cell contains one Flag-labeled htt body (arrow) that is in close proximity to several mitochondria. The nucleus (Nuc) has a few small patches of immunoreactivity.e, Higher magnification of a serial section through the htt body shown in $d$. Note the radiating fibrils in the core (C) and the less defined structure in the shell (S). Multilamellar-type autophagic bodies (a; arrows) abut the shell. $f$, Higher magnification of htt body in $e$. The dashed line is the approximate boundary between the shell $(S)$, which is heavily labeled for Flag, and the core $(C)$, which is not. There are radiating fibrils in the core (arrows). $g$, Higher magnification of the core-shell transition area shown in $f$. Shown are fibrils (arrows) and a globule (arrowhead). $h$, The shell of the htt body has immunoperoxidase-labeled globular structures that associate like beads on a string (arrowheads). Scale bars: $a, d, 5 \mu \mathrm{m} ; b, e 1 \mu \mathrm{m} ; c, f, 0.5 \mu \mathrm{m} ; g, h, 100 \mathrm{~nm}$.

by immunofluorescence microscopy at $\sim 12 \mathrm{hr}$ after transfection, begin to fuse and grow larger and more dense at $\sim 16 \mathrm{hr}$ after transfection, and reach maximal sizes $24-72 \mathrm{hr}$ after transfection (data not shown). Here, we refer to the larger fused htt-labeled structures as htt bodies. The htt bodies also formed in COS1 and MCF-7 cells. COS1 cells proved to have the best transfection efficiency of the three cell lines. COS1 and MCF-7 cells had a larger cytoplasmic volume than the $x 57$ cells, which made it easier in these cells to study the cytoarchitecture of htt bodies by immunofluorescence and electron microscopy (see below). Although a greater proportion of $\mathrm{x} 57$ cells formed htt bodies, MCF-7 cells accumulated the highest density of htt bodies per cell. This ob- servation may be related to the lack of the autophagy regulating protein beclin 1 in MCF-7 cells (Liang et al., 1999).

We used anti-Flag antibody (M5) and different anti-htt antisera [Ab1, EM48, monoclonal antibody (mAb) 2166, and Ab585] to examine htt bodies (Fig. 1A) in MCF-7 cells. There was strong immunoreactivity for Flag, N-htt1-17 (Ab1), htt 414-503 (mAb2166), and htt 585-725 (Ab585) along the perimeter or shell of htt bodies that produced a ring-like labeling (Fig. 1B). The center or core of the htt body was robustly labeled only by antibody EM48. The EM48 polyclonal antibody was made against aa 1-257 of htt without polyglutamine and polyproline domains and detects mutant htt aggregates in cell bodies and 
dendrites in the HD brain (Gutekunst et al., 1999; Li et al., 2001). To confirm the partitioning of different htt epitopes in the shell and core of htt body, double labeling was performed with antibodies mAb2166 and EM48. The results revealed that htt epitopes were segregated within the same htt body (Fig. $1 C$ ). The outermost region of the htt body was labeled by antibody 2166 (Fig. 1C, merged, red), and the core was labeled by EM48 (Fig. 1C, merged, green). An intermediate region was labeled by both antisera (Fig. 1C, merged, yellow).

\section{Electron microscopy of htt bodies}

Htt bodies were examined at the EM level in MCF-7 cells $3 \mathrm{~d}$ after transfection of FH969-18 or FH969-100 (Fig. 2) and immunoperoxidase labeling with Flag antibody. Consistent with the results from immunofluorescence with Flag antibody, htt bodies at the ultrastructural level exhibited Flag immunoperoxidase product heavily along the shell and weaker in the core (Fig. 2a,b). Cells with numerous htt bodies were nearly devoid of organelles (mutant htt) (Fig. 2a). The nuclei of cells enriched with htt bodies contained small foci of Flag labeling but otherwise showed a relatively normal appearance (Fig. 2a).

The core of most htt bodies (both wild type and mutant) contained a mixture of globules, and multivesicular autophagosomes (Fig. 2b,c) (wild type not shown). An additional structure clearly discernable only in htt bodies formed by mutant htt was tortuous fibrils (Fig. 2f,g). Radiating fibrils were prevalent in some of the mutant htt bodies (Fig. $2 f, g$ ). The mutant htt fibrils had an average diameter of $\sim 11.6 \mathrm{~nm}$ and a length of up to 200-250 nm.

The Flag labeling in the shell was associated with globular structures formed with both wt and mutant htt that ranged from $\sim 14$ to $34 \mathrm{~nm}$ in diameter (Fig. $2 \mathrm{~h}$ ). The globules appeared singly, in random clusters, or assembled in a bead-like array that radiated from the core of the htt body (Fig. $2 e, h$ ). The globular assemblies in the shell were the same size and density in htt bodies whether formed by wt (data not shown) or mutant htt. Autophagosomes and mitochondria abutted the htt bodies (Fig. $2 d, e$ ). The membranes of these organelles appeared to fuse with the Flaglabeled globules in the shell (Fig. 1b,e). The EM results supported the findings from immunofluorescence microscopy and showed that the core and shell of htt bodies had different ultrastructures. Also, htt bodies sequestered organelles.

\section{The core is protease resistant, and the shell is protease sensitive}

Expression of the central portion of $\alpha$-synuclein produces filamentous aggregates. The aggregates are resistant to proteinase $\mathrm{K}$ degradation; resistance to degradation serves as a sign of $\alpha$-synuclein insolubility (Miake et al., 2002). We tested htt bodies for resistance to proteinase $\mathrm{K}$. After $72 \mathrm{hr}$ of expression of FH969-100, MCF-7 cells were fixed and treated with different concentrations of proteinase $\mathrm{K}$ and then double labeled using antisera mAb2166 and EM48. Proteinase K treatment $(0.5 \mu \mathrm{g} /$ $\mathrm{ml}$ ) for 15-45 min substantially reduced diffuse htt labeling normally observed in the cytoplasm of transfected cells with either antibody. The shell in many htt bodies was also lost after proteinase $\mathrm{K}$ treatment, whereas the core was preserved (Fig. 3) and frequently enhanced by protease treatment. Densitometry of EM48-labeled htt bodies showed that the mean intensity of the core was significantly increased by treatment for $30 \mathrm{~min}$ with proteinase $\mathrm{K}(0.5 \mu \mathrm{g} / \mathrm{ml})(p<0.0001$; Student's $t$ test; $n=22)$. These results suggested that protease-sensitive and protease-
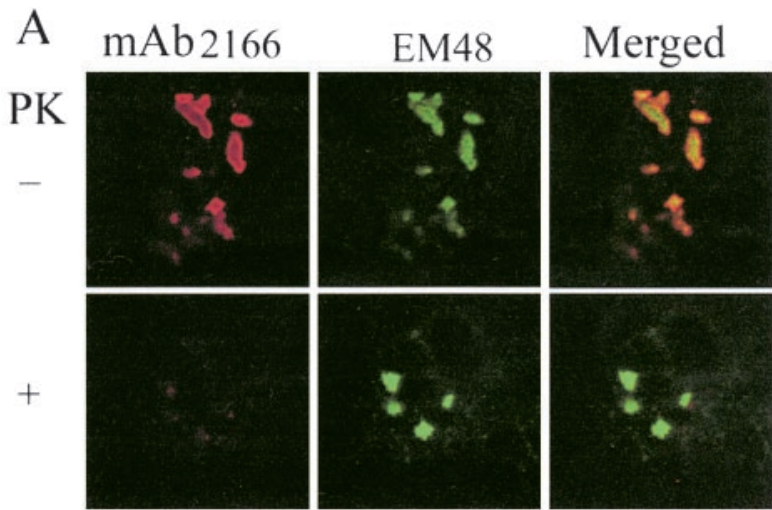

B
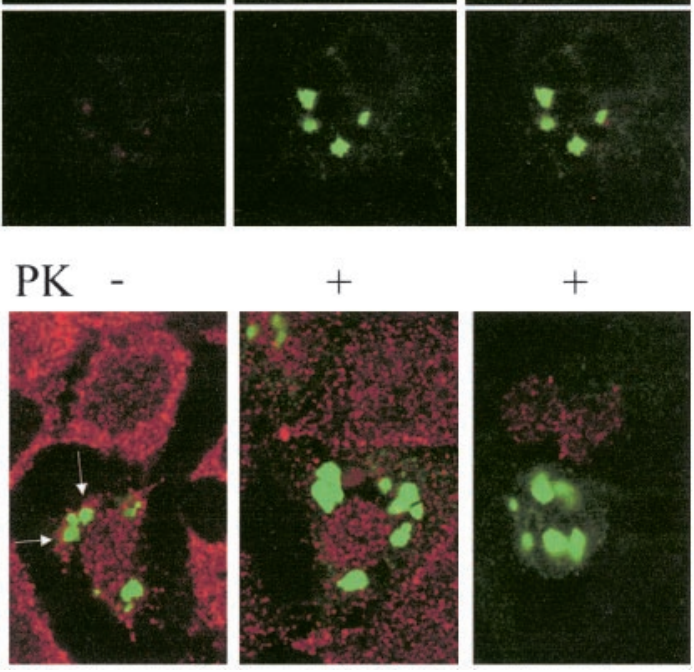

Figure 3. Effects of proteinase K (PK) on htt bodies and dynamin immunoreactivity. MCF-7 cells were transfected with FH969-100, fixed after $24 \mathrm{hr}$, processed with (+) or without (-) PK $(0.25-27 \mu \mathrm{g} / \mathrm{ml})$, as described in Materials and Methods. $A$, One cell without PK treatment (top) and one cell treated with PK (bottom; $0.5 \mu \mathrm{g} / \mathrm{ml}$ for $20 \mathrm{~min}$ ) are representative of results. Double-labeled immunofluorescence of htt bodies with antisera mAb2166 and EM48 reveals that the core (green) is more resistant to protease digestion than the shell (red). PK treatment increased the intensity of labeling within the core. $B$, Merged confocal images of dynamin (red) and htt (detected with EM 48 antibody; green). Left, Dynamin labeling is significantly reduced in the cell with htt bodies compared with the cell above that has no htt bodies. Some dynamin immunoreactivity appears near htt bodies (arrows) and over the nucleus. Middle and right, Images show cells treated with PK $(0.125 \mu \mathrm{g} / \mathrm{ml}$ for $15 \mathrm{~min})$. Note the marked depletion in dynamin immunoreactivity in all cells, including the staining near htt bodies. Mutant htt in the core of the htt body is preserved.

resistant forms of mutant htt were present in the shell and the core of htt bodies, respectively. The core has a compact structure in which the EM48 epitope is partly hidden.

\section{The formation of the shell domain depends on the length} of htt

We looked for critical regions of htt required to form htt bodies, by using cDNAs encoding different lengths of htt. Full-length (1-3144 aa) wt and mutant htt expressed in MCF-7 cells formed fewer htt bodies compared with htt 1-969 and produced more diffuse htt or htt patches in the cytoplasm surrounding htt bodies (Fig. 4). These htt patches are not htt aggregates as formed by smaller N-htt. Expression of FH493-18 produced few cells with htt bodies (data not shown); cells expressing FH400-18 or FH400-100 (data not shown) and FH287-18 or FH287-100 formed no htt bodies (Fig. 4). Perinuclear aggregates (with no shell) appeared in some cells transfected with FH287-100, and labeling of htt occurred in the cytoplasm, nucleus, and plasma membrane of cells transfected with FH287-18Q (Fig. 4). These results suggested that in vitro assembly of htt bodies from wt or mutant htt required sequence of htt larger than or equal to aa 493.

Htt bodies alter the distribution of vesicle-associated proteins Wild-type or mutant htt codistributes with clathrin-coated membranes in fibroblasts (Velier et al., 1998). These findings 


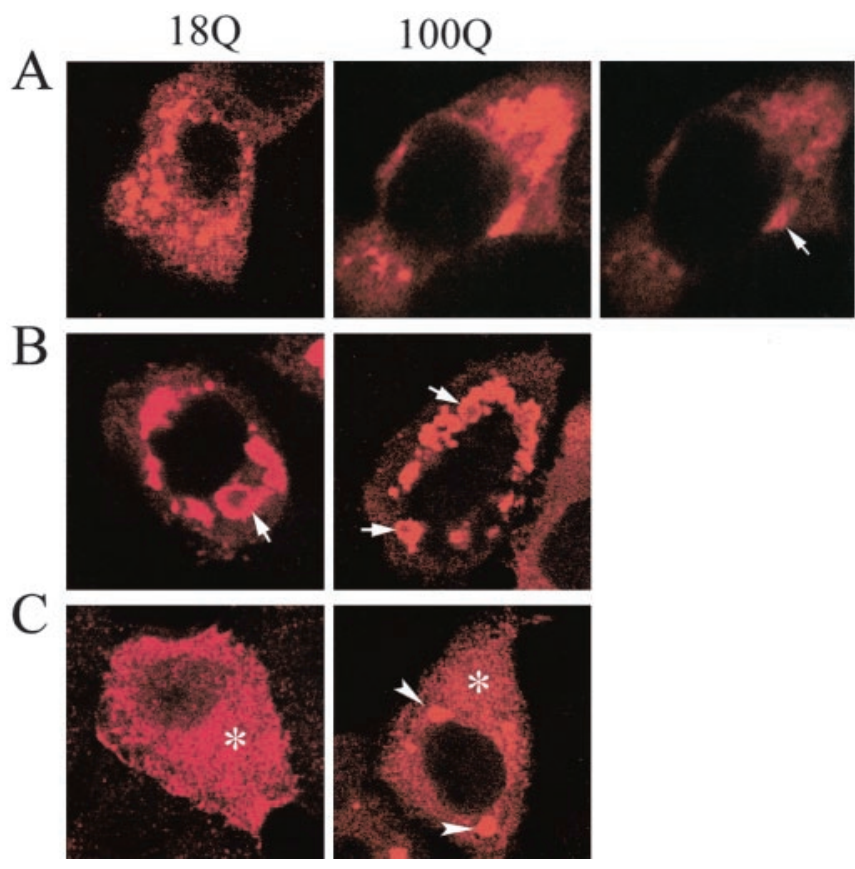

Figure 4. The formation of htt bodies is effected by the size of htt. MCF-7 cells were transfected with wt (180) or mutant (100Q) FH3144 (A), FH969 (B), and FH287 ( $C$ and processed for immunofluorescence with anti-Flag antibody (M5) $24 \mathrm{hr}$ after htt transfection, as described in Materials and Methods. Full-length htt expression $(A)$ produces htt bodies, but they are less developed and there is more diffuse htt labeling. The adjacent panel with lighter exposure shows labeling of shell (arrow) of htt body. Numerous htt bodies with characteristic labeling of the shell region (at arrows) appear when FH969-180 or FH969-100Q is used for transfection. A few mutant htt aggregates appear with FH287-100Q (C, arrowheads), but htt remains diffusely dispersed in the cytoplasm ${ }^{*}$ ) and plasma membrane when expressed from FH287-180.

support a role for wt htt in endocytosis and suggest that mutant htt could disrupt endocytic function. Dynamin functions in endocytosis by regulating the assembly of clathrin-coated vesicles (Sever, 2002). Endogenous dynamin immunoreactivity occurred at plasma membranes and intracellular vesicles in MCF-7 cells (Fig. $3 B$ ) and clonal striatal cells (results not shown). In cells with htt bodies, dynamin labeling was markedly reduced at intracellular and plasma membranes (Fig. 3B). Some dynamin staining occurred at htt bodies and the nuclear membrane (Fig. 3B). HIP1 is an actin-binding protein involved in endocytosis (EngqvistGoldstein et al., 1999; Metzler et al., 2001; Waelter et al., 2001). HIP1 localizes to plasma membranes (Metzler et al., 2001; Waelter et al., 2001). In cells with htt bodies, the localization of endogenous HIP1 was substantially reduced at plasma membranes and partly redistributed to htt bodies. SH3GL3 and FIP2 (a tumor necrosis factor- $\alpha$-inducible coiled-coil protein) also function in membrane transport and interact with htt (Sittler et al., 1998; Hattula and Peränen 2000; So et al., 2000; Sever, 2002). In cells with htt bodies, labeling for these proteins was reduced at normal membrane locations and occurred in the shell of htt bodies (Fig. 5). SH3GL3 and FIP2 showed greater localization to htt bodies than dynamin and HIP1. DLP1 is involved in maintaining the localization and function of mitochondria (Pitts et al., 1999) and normally displays a punctate distribution throughout the cytoplasm. In cells with htt bodies, DLP1 immunoreactive structures were relocated near htt bodies but did not colocalize with htt bodies (Fig. 5). Other proteins that were partly redistributed to the shell of htt bodies included active caspase-3, which was found in $\sim 50 \%$ of cells with htt bodies, and proteasome and HSP70, which were present in all htt bodies (Table 1).
We questioned whether the dynamin immunoreactivity associated with htt bodies was affected by treatment with proteinase K. Double-label immunofluorescence was performed in cells transfected with FH969-100Q and treated for 15 to 30 min with $0.125-0.5 \mu \mathrm{g} / \mathrm{ml}$ proteinase $\mathrm{K}$ treatment. Antibody EM48 was used to label the core of htt bodies. Proteinase K treatment reduced, in a dose-dependent manner, dynamin immunoreactivity within the cytoplasm. Dynamin labeling associated with htt bodies was reduced by proteinase $\mathrm{K}$ treatment for $15 \mathrm{~min}$ at the lowest concentrations (Fig. $3 B$ ). In contrast, the core of htt bodies, as detected with EM48 antibody, was highly resistant to proteinase $\mathrm{K}$ treatment with all treatment conditions. These results suggested that the dynamin near htt bodies was protease sensitive.

The core had different and fewer detectable immunoreactive proteins than the shell. As reported previously in clonal striatal cells (Kegel et al., 2000), the lysosomal enzyme cathepsin D was concentrated in the core of htt bodies (Table 1) and accumulated to a greater extent when htt bodies were formed by mutant htt than wt htt (FH969-100 vs FH969-18). Ubiquitin and HSP40 also appeared in the core (Table 1); levels of these two proteins were not different for htt bodies formed by wt and mutant htt.

HSP60 localizes to mitochondria and was not found in htt bodies (Table 1). In cells with htt bodies, HSP60-positive mitochondria were removed from their normal location throughout the cytoplasm and concentrated around and between htt bodies.

\section{Association between formation of htt bodies and diminished transferrin uptake}

As a functional test of membrane transport, we measured internalization of transferrin by the endocytic pathway. Approximately $95 \%$ of untransfected MCF-7 cells internalized and transported rhodamine-labeled transferrin within $30 \mathrm{~min}$. The fluorescence was punctate and dispersed throughout the cytoplasm (Fig. 6A, top). Within the same culture dish, there was no difference in the intracellular distribution of labeled transferrin among transfected cells expressing wt or mutant Flag-htt diffusely in the cytoplasm and cells without apparent Flag-htt expression (Fig. $6 \mathrm{~A})$. A significantly greater proportion $(\sim 30 \%)$ of cells with htt bodies had little or no transferrin labeling compared with cells with no Flag-htt expression or cells with only diffuse Flag-htt labeling ( $\sim 3-8 \%$; $p<0.05$; Student's $t$ test; $n=4$ ) (Fig. $6 A, B)$. There was no difference in the extent of transferrin incorporation between cells with htt bodies formed by wt htt and mutant htt.

\section{Polyglutamine and polyproline regions determine the formation of htt bodies and redistribution of cytoplasmic proteins}

We examined the effects of polyglutamine expansion on the frequency of htt bodies in MCF-7 cells. Results showed that the number of cells with htt bodies was significantly greater after expression of FH969-100 than FH969-18 ( $p<0.001 ; n=6$; Student's $t$ test) (Fig. 7A). The percentage of cells with htt bodies increased with time after transfection of FH969-100. Seventytwo hours after transfection, $>40 \%$ of cells transfected with FH969-18 or FH969-100 had htt bodies. At all time points examined (6-72 hr), cells expressing mutant htt with 100Q had more htt bodies (ANOVA; $F=38.93$; $p<0.05$ ) (Fig. 7A; bar figure not shown). Expression of FH969-46 produced more htt bodies than expression of FH969-18, but the difference between mutant and wt htt was not statistically significant.

Proline-rich regions play an important role in signaling path- 


\section{Non-transfected Transfected with FH969-100}
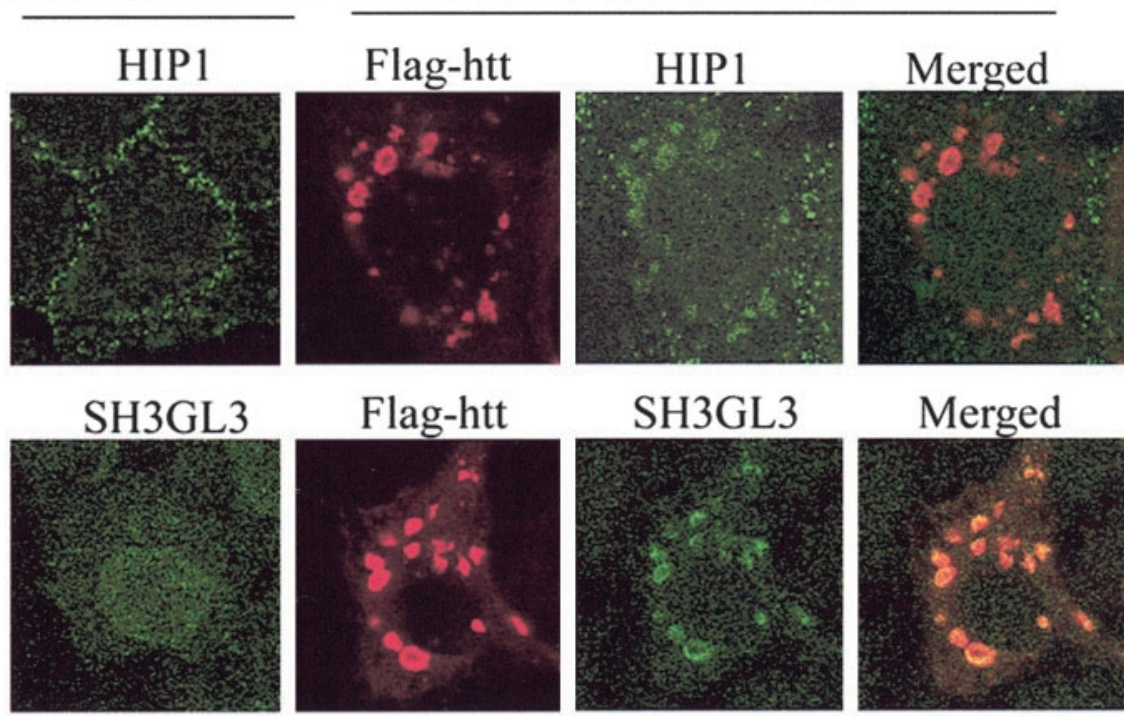

FIP2

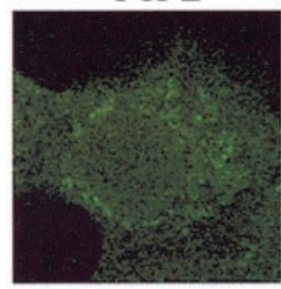

Flag-htt
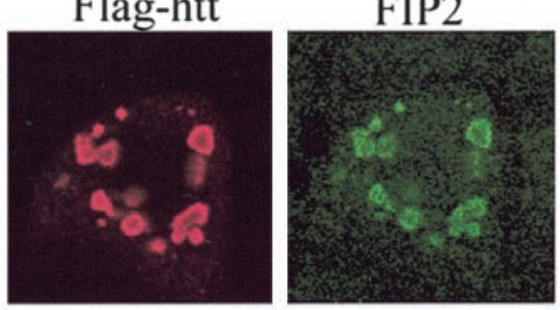

Dynamin

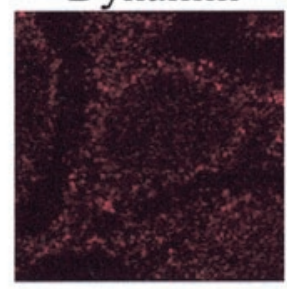

Flag-htt
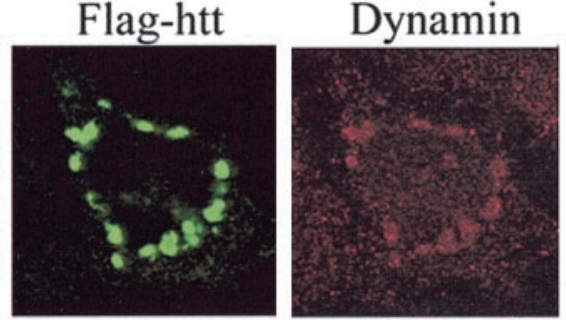

DLP1
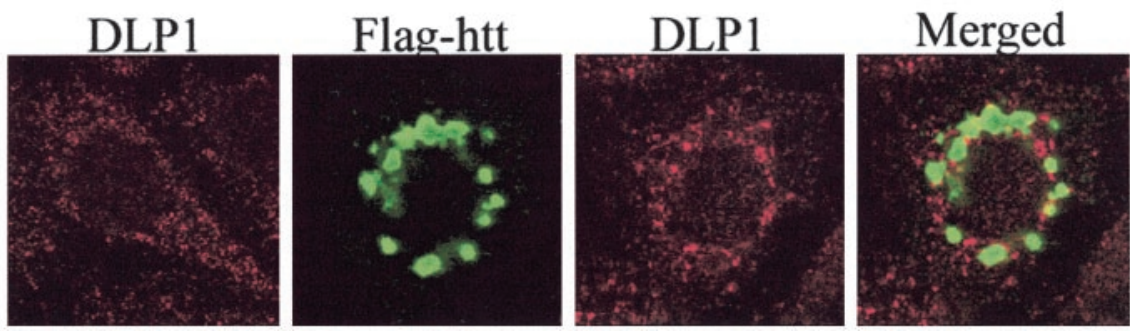

Figure 5. Colocalization of cytoplasmic proteins with htt bodies. MCF-7 cells were transfected with truncated FH969-100. Cells were fixed and processed for double-label immunohistochemisry $24 \mathrm{hr}$ after htt transfection, as described in Materials and Methods. Cells were analyzed with a confocal microscope, and images were merged in Adobe Photoshop. Flag-htt is identified with anti-Flag antibody M5. HIP1, SH3GL3, dynamin, and FIP2 colocalized to the periphery of htt bodies, altering their normal localization. DLP1 redistributed to the regions adjacent to $h$ tt bodies.

ways that regulate membrane trafficking associated with autophagy and endosomal/lysosomal functions (Klionsky, 1999). The polyproline region in $\mathrm{htt}$ is required for some protein interactions including interaction with SH3GL3 (Liu et al., 1997; Sittler et al., 1998). Therefore, we speculated that removing the proline region in htt might affect the formation of htt bodies or the redistribution of cytoplasmic proteins to htt bodies. To test these possibil-

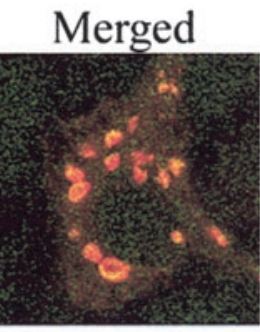

Merged

ities, FH969-18 proline - and FH969-46 proline - were expressed in clonal striatal cells (x57) or MCF-7 cells. Subcellular fractions of cell lysates were examined by SDS-PAGE and Western blot. Removal of the proline-rich region in htt from aa 41-89 reduced the size of the Flag-htt fusion protein seen on Western blot, as expected. Polyproline deletion did not change levels of intact htt expressed in different subcellular fractions (Fig. 7B), except for a small decrease in the P1 fraction, which contains nuclei and nuclear and perinuclear membranes. The number of cells that formed htt bodies was greater in clonal striatal cells than in MCF-7 cells (Fig. 7C). In both cell lines, polyproline removal in htt significantly reduced the number of cells with htt bodies. The number of cells with htt bodies was reduced by $\sim 80 \%$ in cells expressing FH969-18 proline - and by $40 \%$ in cells expressing FH969-46 proline $-(46 \mathrm{Q} ; p<0.05 ; n=$ 6; Student's $t$ test) (Fig. $7 C$ ). The results suggested that the proline-rich region in $\mathrm{htt}$ is involved in forming htt bodies. In these studies, 46 polyglutamines were used for mutant htt because of technical difficulties in making a deletion of the prolinerich region with 100 glutamines.

Deletion of the proline-rich domain in htt changed the architecture of htt bodies. Polyproline removal abolished the partitioning of htt epitopes into a shell and core that was normally seen with antibodies mAb2166 and EM48, respectively (Fig. $7 D)$. Instead, these anti-htt antisera produced immunofluorescent labeling that largely codistributed in patches. In some cells, the patches were smaller and more dispersed than htt bodies (Fig. 7D).

Removing the polyproline region reduced or eliminated labeling for HIP1, SH3GL3, dynamin, and proteasome in the shell of htt bodies (Fig. 8). In contrast, the colocalization of HSP40, HSP70, and ubiquitin with Flag was still found in the htt-labeled patches that formed after deletion of the proline-rich region (data not shown). These results implicate the polyproline region in htt in interactions with proteins involved in vesicle trafficking. The presence of the polyproline region facilitates sequestration of these proteins, which can lead to changes in membrane function.

Immunoreactive dynamin accumulates in cytoplasmic bodies in grade $1 \mathrm{HD}$ cortical neurons

In cells with htt bodies, dynamin was absent from intracellular and plasma membranes and occurred near htt bodies and over the nucleus. We used the immunoperoxidase method to look for dynamin labeling in the cortex of control and HD brains. In 
Table 1. Codistribution of htt epitopes and cytoplasmic proteins with the shell and core of htt bodies

\begin{tabular}{lll}
\hline Region of htt body & htt epitopes & Other proteins \\
\hline Shell & Ab1 (aa 1-17) & HIP1 \\
& mAb2166 (aa 414-503) & SH3GL3 \\
& Ab585 (aa 585-725) & FIP2 \\
& Dynamin \\
& & Active caspase-3 \\
& Proteasome \\
& & HSP40 \\
& & HSP70 \\
& EM48 (aa 1-257) & Cathepsin D \\
Core & Ab1 (weak staining) & Ubiquitin \\
& & HSP40 \\
\hline
\end{tabular}

control brain, most pyramidal neurons displayed low levels of diffuse cytoplasmic labeling for dynamin (Fig. 9a). In HD grade 1 (Fig. $9 b, d, e$ ) and grade 3 (Fig. $9 c$ ) cortex, the majority of neurons showed a marked increase in dynamin staining, especially in perinuclear regions where the immunoreactivity was concentrated in spherical bodies. Increasing the contrast in the digital images helped to visualize the dynamin-labeled bodies (Fig. 9d,e) and, in some cases, revealed more labeling along the periphery than in the center of these structures (Fig. $9 d$ ). The localization of dynamin in HD neurons is reminiscent of dynamin labeling in the shell of mutant htt bodies in vitro. Other sections were labeled with antibody EM48, which preferentially detects mutant htt aggregates in the HD brain (Gutekunst et al., 1999). There was no staining in control cortex with EM 48 antibody (results not shown). In the grade $1 \mathrm{HD}$ brains, some cortical neurons displayed intense labeling for mutant htt in the nucleus and in perinuclear aggregates (Fig. 9f), which had a size comparable with htt bodies observed in vitro. These results suggest that, in early stages of HD, dynamin redistributes to mutant htt bodies in cortical neurons.

\section{Discussion}

$\mathrm{N}$-terminal fragments of mutant htt expressed from exon 1 (1-89 aa of wt) accumulate in vitro and in mice and form insoluble aggregates in nuclear and perinuclear regions of neurons (Davies et al., 1997; Scherzinger et al., 1997). The aggregates have a fibrillar, granular morphology similar to those seen in the HD brain (DiFiglia et al., 1997). The role of aggregates in HD pathogenesis is unclear; their presence in some HD cell and animal models inconsistently correlates with cell death (Saudou et al., 1998; Kuemmerle et al., 1999). A nonfibrillar soluble form of mutant htt could engage in abnormal protein interactions, thereby contributing to HD pathology (Passani et al., 2000; Gervais et al., 2002). In this study, we sought evidence of abnormal interactions by examining the htt body, which forms in cultured cells ectopically expressing a large $\mathrm{N}$-terminal fragment of wt or mutant htt. Polyglutamine expansion increased the formation of htt bodies. The htt bodies develop from the fusion of htt-enriched autophagosomes (Kegel et al., 2000). We found that a protease-sensitive htt accumulated in globular assemblies at the periphery of htt bodies and colocalized with cytoplasmic proteins involved in vesicle trafficking. The presence of htt bodies hindered cellular endocytic uptake of transferin. In addition, numerous multivesicular membranes, autophagosomes, and mitochondria were incorporated into or adherent to the surface of htt bodies. The mitochondria may be damaged by htt and targeted for removal by autophagosomes. Recently, Panov et al. (2002) proposed that direct association of mutant htt with mitochondrial membranes caused calcium dysregulation in HD mitochondria. Altogether, these findings raise the possibility that an excess of soluble mutant htt that accumulates in the cytoplasm of neurons may contribute to HD pathogenesis.

The depletion of dynamin, HIP1, FIP2, and SH3GL3 in cells with htt bodies supports the possibility that there is altered function of vesicle movement in HD pathogenesis (DiFiglia et al., 1995). These proteins contribute to the assembly of large protein complexes that are involved in regulating vesicle trafficking and membrane flow (Sittler et al., 1998; Wanker et al., 1999; Hattula and Peränen, 2000; Sever, 2002). The displacement of these proteins from their normal site by htt bodies is likely to disturb functions associated with vesicle trafficking and endocytosis. We found that the uptake or distribution of transferrin in many cells with htt bodies was severely impaired, evidence that membrane transport is affected. Dynamin, which functions in clathrinmediated endocytosis (Sever, 2002), is redistributed to perinuclear regions in HD cortical neurons of grade 1/presymptomatic HD patients. Grade $1 \mathrm{HD}$ neurons also show build up of mutant htt in cytoplasmic bodies (present study; Gutekunst et al., 1999). Modregger et al. (2002) showed that PASCIN1, a protein involved in synaptic vesicle recycling, interacts with htt and is depleted from HD neuronal processes and accrues in perinuclear locations with mutant htt in presymptomatic HD brains. These findings suggest that dysfunction in membrane transport may occur at early stages of HD.

Cytoplasmic protein redistribution to htt bodies was not changed by polyglutamine expansion from 18 to 46 or 100 glutamines. This suggests that the polyglutamine region is not sufficient to regulate the sequestration of soluble proteins into htt bodies. Removal of the polyproline region in htt reduced htt bodies and their capacity to recruit HIP1, SH3GL3, FIP2, and dynamin. Our findings support an important role of the polyproline region in normal htt protein interactions. In our study, the lack of proline-rich region in wt htt reduced htt bodies much more than proline deletion in mutant htt. It may be that polyglutamine expansion interferes with the regulation or stability conferred by the proline region in htt. The polyproline region is also critical for aggregation of mutant htt. Khoshnan et al. (2002) found that an intrabody directed to the proline region in htt reduced mutant htt aggregates in vitro. Although not studied here, the HEAT (for huntingtin, elongation factor 3, regulatory A subunit of protein phosphatase $2 \mathrm{~A}$ and TOR1) repeat sequences in the $\mathrm{N}$ terminus of htt may also be important in targeting htt to autophagosomes and forming htt bodies. These tandem repeats of specific amino acid residues occur in other proteins that have some role in membrane transport (Andrade and Bork, 1995; Takano and Gusella, 2002). For example, HEAT motifs have been found to mediate the membrane localization of Tor2p, a component of a signaling pathway that regulates cell growth and autophagy in yeast (Kunz et al., 2000).

Our results show that polyglutamine expansion in htt is not required for htt to sequester cytoplasmic proteins away from their normal location in the cytoplasm. High levels of wt htt are capable of recruiting cytoplasmic proteins. In other studies, we also found that N-terminal fragments of wt htt 1-287 or 1-400 form nuclear inclusions, as does mutant htt in vitro (Y. J. Kim and M. DiFiglia, unpublished observations). Transient transfection of htt in vitro, as used in the present study, is conducive to observe these characteristics of wt htt. There is precedence for toxicity of wt proteins with polyglutamine tracts. Expression of high levels of fulllength wt ataxin 1, another polyglutamine-containing protein, 
A
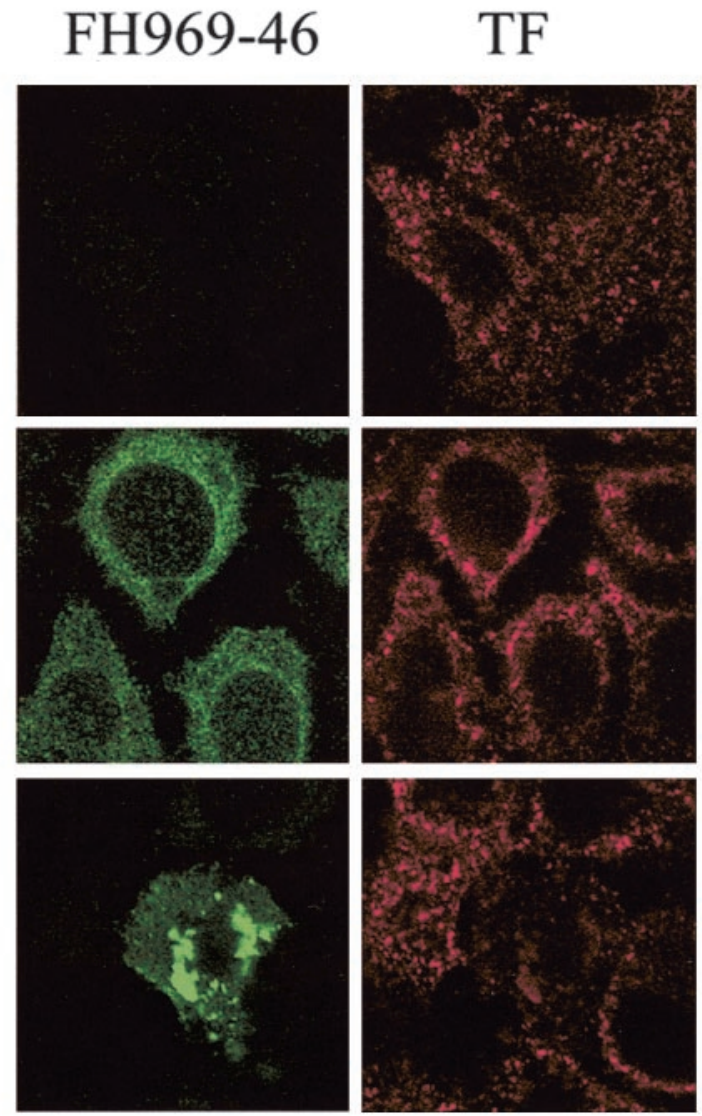

B

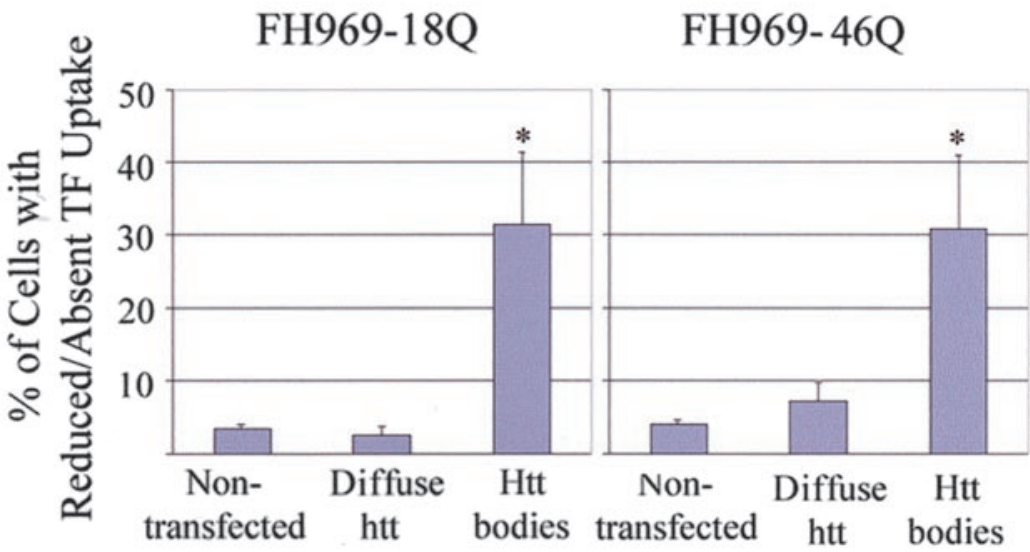

Figure 6. Effects of htt bodies on uptake of transferrin. A, MCF-7 cells from the same culture dish were treated with FH969$46 \mathrm{Q}$ for $24 \mathrm{hr}$ and incubated with rhodamine-labeled transferrin (TF) at $37^{\circ} \mathrm{C}$ for the last $30 \mathrm{~min}$. Immunofluorescent Flag labeling (green) is absent from some cells (top left) or is present as a diffuse product (middle left) or in htt bodies (bottom left). TF incorporation (red) is similar in cells without Flag-htt expression or diffuse Flag-htt expression but is markedly diminished in the cell with htt bodies (bottom left). B, Percentage of cells without Flag-htt expression or with Flat-htt expression (diffuse or in htt bodies) that had little or no TF uptake. Approximately one-third of cells with htt bodies formed by either wt or mutant htt were significantly impaired in their capacity to take up or transport transferrin compared with nontransfected cells. Five hundred Flag-positive cells were analyzed on each slide. Bar, mean \pm SE. ${ }^{*} p<0.05$ (Student's $t$ test; $n=4$ slides). the availability of HIP1. An elevation of HIP1 was toxic to cells in vitro, suggesting a mechanism for HD pathogenesis. The results from both studies suggest that two mechanisms, a polyproline-dependent one and a polyglutamine dependent one, differentially modulate the levels of HIP1 in HD neurons.

Mutant htt bodies have an amalgam of morphological features characteristic of cytoplasmic aggregates (fibrillar structure) and autophagosomes (multivesicular bodies and cathepsin D immunoreactivity). The size of the fibrils in mutant htt bodies $(11.6 \mathrm{~nm})$ is similar to the size of the fibrils identified in aggregates formed by HD exon 1 and other aggregate-prone proteins (Scherzinger et al., 1997; Bevivino and Loll, 2001; Perutz et al., 2002; Poirer et al., 2002). The presence of fibrillar structure characteristic of aggregates found in HD autopsy brains indicates that the formation of htt bodies does not depend on overexpressing htt, although it may require accumulation. Polyglutamine expansion increased the frequency of cells that formed htt bodies (present study) and caused more cathepsin D to accumulate in the core (Kegel et al., 2000). Cathepsin D is transported to maturing autophagosomes. The multivesicular bodies within the core of the htt body may be the sites of cathepsin D accumulation. The htt bodies are also regulated by autophagy. Treating cells with the autophagy inhibitor 3 -methyladenine significantly reduced the frequency (Qin et al., 2003).

The formation of the shell of the htt body minimally requires an $\mathrm{N}$-terminal region of htt that terminates between aa 493 and 969. N-htt fragments in this size range are present in protein extracts from the HD brain and may be partly formed from cleavage by caspases and calpains (Kim et al., 2001; Gafni and Ellerby, 2002; Wellington et al., 2002). HIP1, which was redistributed to the shell, binds to a region in htt between aa 222-480 (Michael R. Hayden, personal communication) (i.e., within the size of htt required to form htt bodies. In contrast, the core of the htt body may contain $\mathrm{N}$-htt fragments that are smaller than those in the shell. Only $\mathrm{N}$-terminal anti-htt antibodies Ab1 and EM48 reacted with the core.

Polyglutamine expansion in htt incaused neurodegeneration in a Drosphila model of spinocerebellar ataxia (Fernandez-Funez et al., 2000). Our study showed that polyglutamine expansion increased the number of cells forming htt bodies. However, relocation of interacting proteins, including HIP1, to $\mathrm{htt}$ bodies was polyproline dependent and polyglutamine independent. Gervais et al. (2002) showed that polyglutamine expansion in $\mathrm{htt}$ reduced the interaction between htt and HIP1, which increased creases the probability that partially unfolded intermediate forms will self-associate as oligomers and evolve into a fibrillar structure (Perutz et al., 2002). The protofibril has been identified as a stable soluble prefibrillar intermediate of many aggregate prone proteins, including mutant htt (Perutz et al., 2002). Poirer et al. (2002) used transmission EM and atomic force microscopy to examine purified HD exon 1. They identified small globules of 

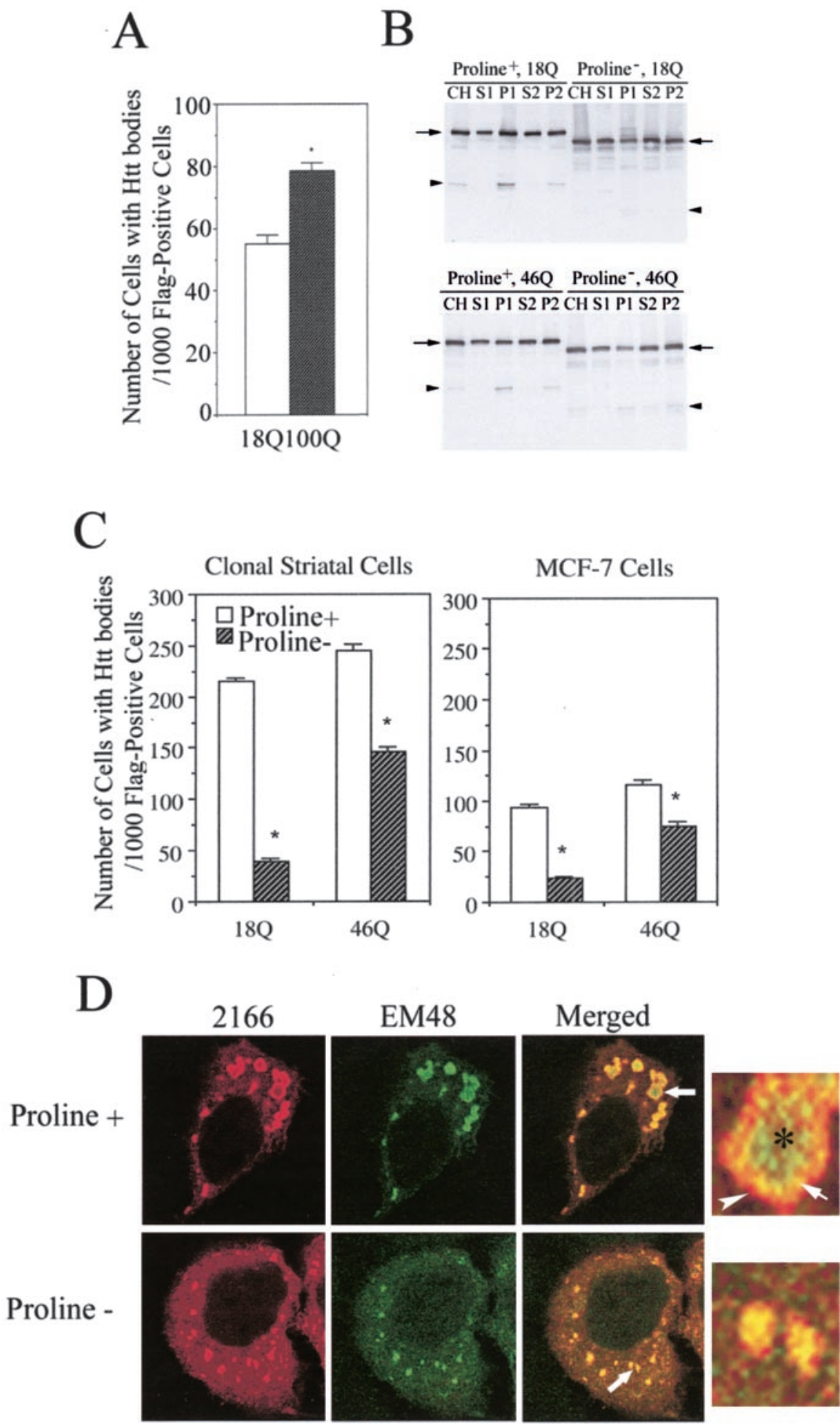

Figure 7. Effects of polyglutamine and polyproline regions on the formation of htt bodies in MCF-7 cells and clonal striatal cells (x57). A, The number of MCF-7 cells with htt bodies per 1000 Flag-positive cells is significantly increased in cultures transfected with FH969-100 compared with cultures transfected with FH969-18. One thousand Flag-positive cells were analyzed on each slide. ${ }^{*} p<0.05$ (Student's $t$ test; $n=6$ slides). B, Western blots of htt expression in subcellular fractions prepared from cells transfected with FH969-18 and FH969-46 with ( + ) and without $(-$ ) the encoded polyproline region. Levels of intact htt (arrows) are approximately the same after polyproline deletion in the crude homogenate (CH), soluble (S1, S2) and membrane (P2) fractions. The P1 fraction shows a small decline in htt levels after proline deletion. Proline deletion changes the size of Flag-htt based on its faster migration on SDS-PAGE. N-htt fragments (arrowhead) still form after polyproline deletion, although proline removal in wt htt alters the pattern of breakdown products. C, x57 and MCF-7 cells were transfected with FH969-18 or FH969-46 with or without the polyproline region in htt, and the formation of htt bodies was analyzed $24 \mathrm{hr}$ after transfection as in A. Note that a greater proportion of clonal striatal cells form htt bodies than MCF-7 cells. There is a significant reduction in the number of cells that form htt bodies when the polyproline region is removed (proline - ) from wt or mutant htt in both cells lines (Student's $t$ test; ${ }^{*} p<0.05$ ). $D$, Immunofluorescence labeling for htt bodies in MCF-7 cells with antibodies 2166 and EM48 reveals changes in the morphological appearance of htt-labeled dense structures when the polyproline region is removed (proline-). With the intact polyproline region (proline +) in htt, htt epitopes in htt bodies segregate into a shell (red, arrowhead) and core (green, asterisk) and overlap in an intermediate region (yellow, arrow) as shown in the enlarged htt body from the merged image and also in Figure 1C. With polyproline deletion (proline-), more diffuse htt occurs in the cytoplasm, and htt epitopes do not partition into shell and core domains but codistribute in patches (see enlargement from merged image). Some patches are smaller than htt bodies. 

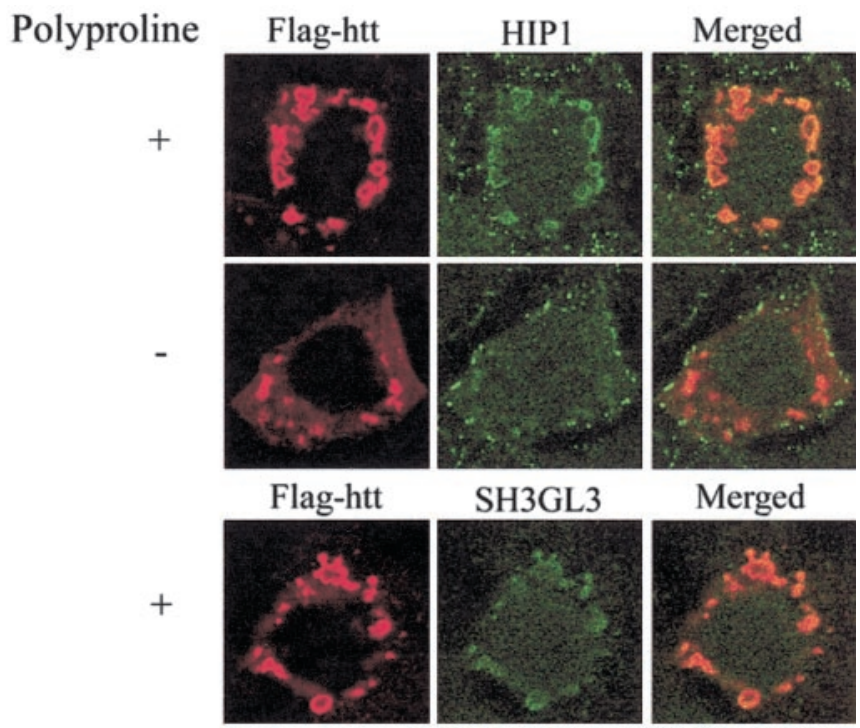

SH3GL3
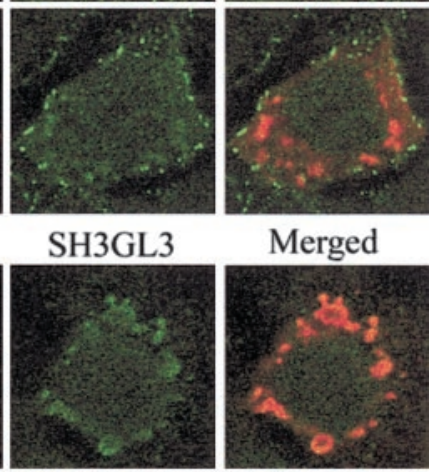

Merged
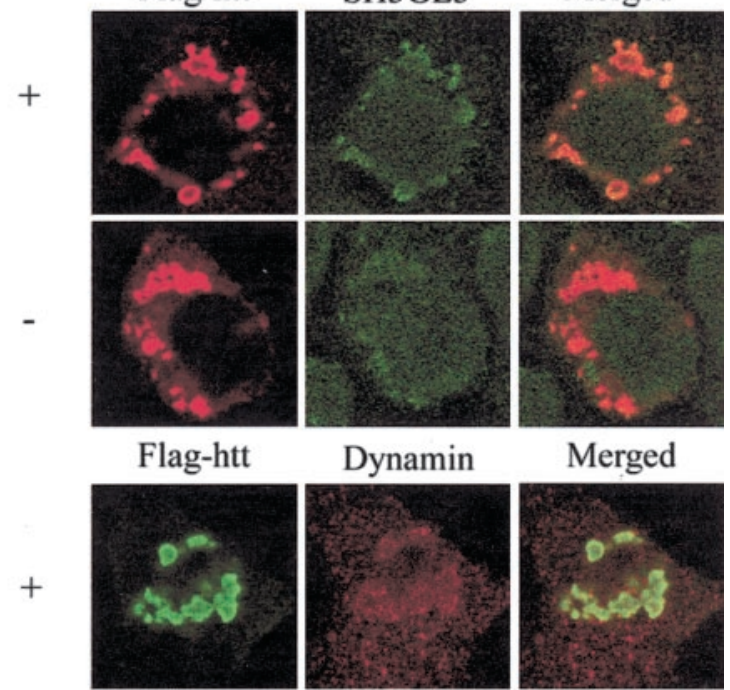

Dynamin
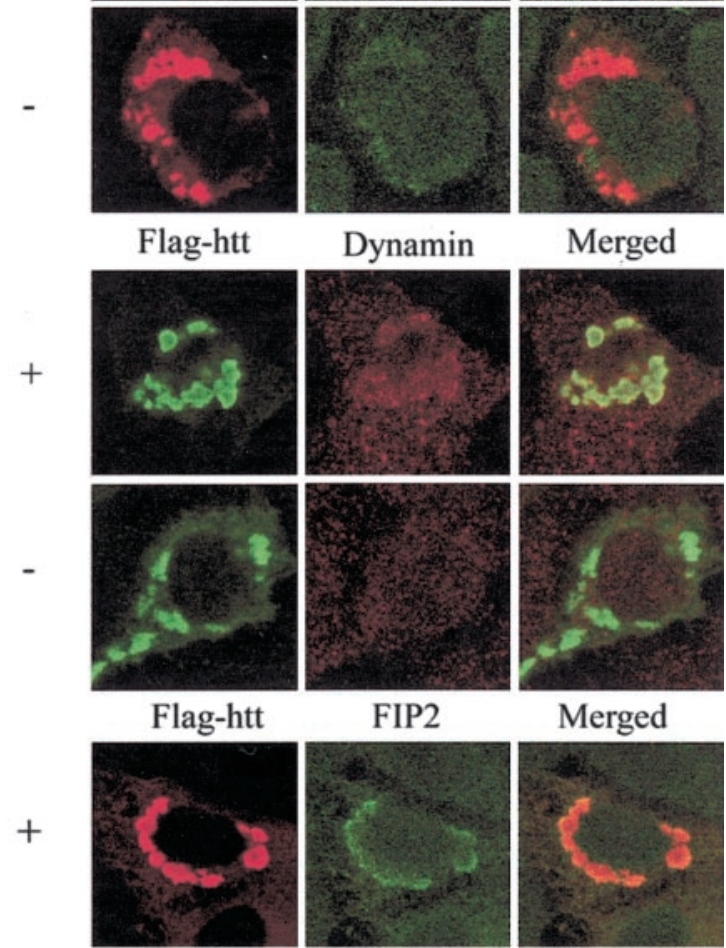

FIP2

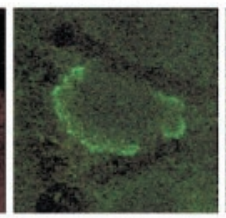

Merged
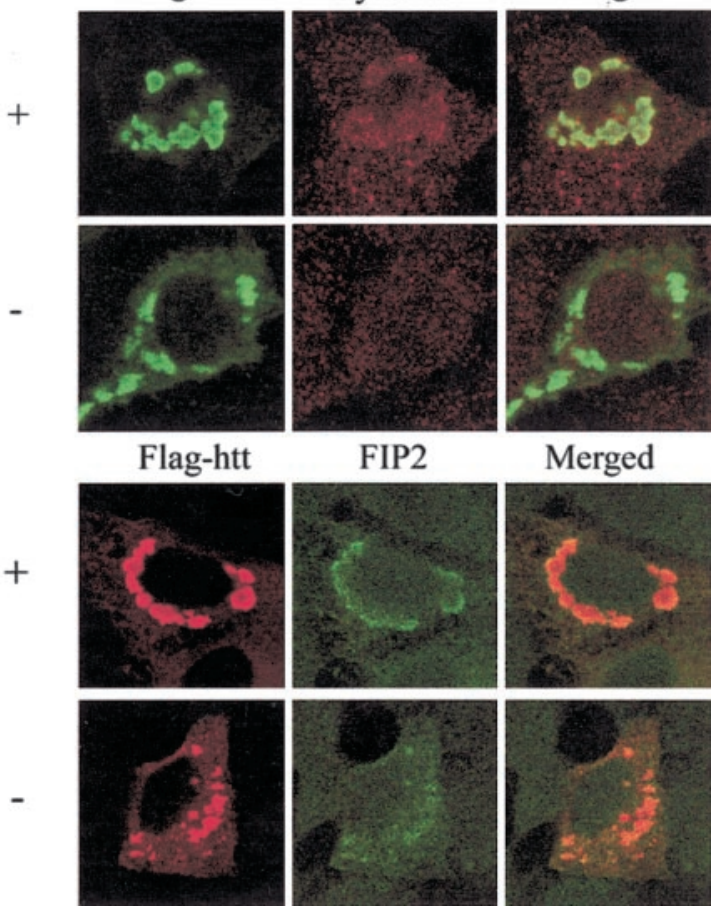

Figure 8. Effects of polyproline deletion on relocation of HIP1, SH3GL3, dynamin, and FIP2. MCF-7 cells were transfected with FH969-18 or FH969-46 with polyproline region intact $(+)$ or removed ( - ). Cells were fixed and processed for immunohistochemisry $24 \mathrm{hr}$ after transfection, as described in Materials and Methods. Cells were analyzed with a confocal microscope, and images were processed with Adobe Photoshop. After polyproline deletion, the shell region of htt bodies is absent, and colocalization of HIP1, SH3GL3, dynamin, and FIP2 with htt bodies is eliminated or substantially reduced.

$\sim 4-5 \mathrm{~nm}$ as the oligomers of mutant htt that form protofibrils, a structure they compared in appearance to "beads on a string." Two protofibrils were predicted to form the mature fibril. The globules we found in the shell of htt bodies in cells also assembled like beads on a string but were significantly larger $(\sim 14-34 \mathrm{~nm})$
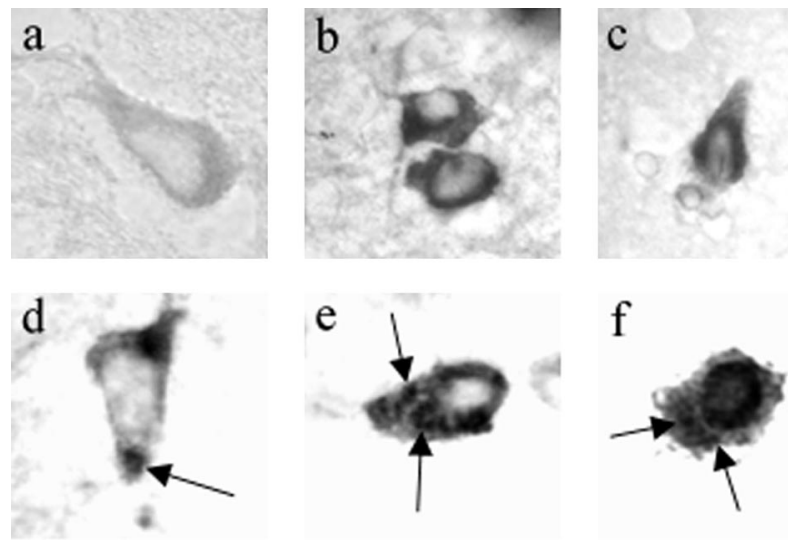

Figure 9. Dynamin immunoreactivity in control and HD brain. Immunoperoxidase labeling for dynamin is shown in cortical neurons from control brain $(a)$ and HD brain $(b-e)$. There is a marked redistribution of dynamin in $\mathrm{HD}$ grade $1(b, d, e)$ and $\mathrm{HD}$ grade $3(c)$ neurons. Intense immunoreactivity appears in perinuclear regions and on cytoplasmic bodies ( $d, e$, arrows). The staining within nuclei in $b$ and $c$ is cytoplasmic staining within nuclear invaginations. In $f$, mutant htt labeling detected with EM48 antibody is shown in HD grade 1 cortex. Mutant htt occurs in the nucleus and in cytoplasmic bodies (arrows). In $d-f$, contrast was enhanced to show cytoplasmic bodies.

than those identified by Poirer et al. (2002). Recently, Bitan et al. (2003) reported that $\beta$-amyloid forms large beaded super structures of $20-50 \mathrm{~nm}$ in vitro in addition to the $10 \mathrm{~nm}$ diameter protofibrils. The large beaded globules were identified by them as higher-order oligomers (Bitan et al., 2003). It was not clear from their studies whether the large super structures were involved in the formation of fibrils. We speculate that the $14-34 \mathrm{~nm}$ spherical bodies prevalent in the shell of htt bodies are oligomers of htt. The native full-length htt has been predicted to exist as a dimer (Bao et al., 1996); oligomers of higher order have not been reported. We cannot rule out from our analysis that htt monomers also occur in htt bodies. Biochemical analysis of htt bodies isolated from cultured cells will be necessary to determine the predominant forms of htt in htt bodies.

Wild-type and mutant htt bodies contained proteins involved in protein folding. Ubiquitin and HSP40 were present in the core and proteasome, HSP40, and HSP70 accumulated in the shell. These observations suggest that the core and shell of htt bodies contain misfolded species of htt targeted for removal or degradation. It was surprising that ubiquitin and proteasome were segregated in the core and shell, respectively, because these proteins work in concert to degrade misfolded proteins and would be expected to colocalize. The ubiquitin may be associated with htt in multivesicular bodies in the core of htt bodies. Targeting of some proteins to multivesicular bodies requires ubiquitination (Katzmann et al., 2002). An intact polyproline region in htt was required for recruiting proteasome, indicating a possible direct interaction between proteasome and the polyproline region of htt.

In summary, this study finds that exogenous expression of large truncated wt and mutant htt formed htt bodies that had structurally distinct shell and core regions. A soluble multioligomeric htt assembled in the shell and colocalized with other proteins involved in vesicle trafficking that were redistributed away from their normal site of function. The presence of htt bodies depleted and displaced organelles and impaired endocytic pathways. Because mutant htt forms more htt bodies than wt htt, these effects are more severe in the cells expressing mutant htt. We propose that, in HD neurons, a soluble oligomeric assembly of mutant htt similar to that found in the shell of the htt body 
contributes to $\mathrm{HD}$ pathogenesis by disrupting membrane trafficking.

\section{References}

Andrade MA, Bork P (1995) HEAT repeats in the Huntington's disease protein. Nat Genet 11:115-116.

Bao J, Sharp AH, Wagster MV, Becher M, Schilling G, Dawson CA, Dawson M (1996) Expansion of polyglutamine repeat in huntingtin leads to abnormal protein interactions involving calmodulin. Proc Natl Acad Sci USA 93:5037-5042.

Bevivino AE, Loll PJ (2001) An expanded glutamine repeat destabilizes native ataxin-3 structure and mediates formation of parallel $\beta$-fibrils. Proc Natl Acad Sci USA 98:11955-11960.

Bitan G, Kirkitadze MD, Lomakin A, Vollers SS, Benedek GB, Teplow DB (2003) Amyloid $\beta$-protein (A $\beta$ ) assembly: $A \beta 40$ and $A \beta 42$ oligomerize through distinct pathways. Proc Natl Acad Sci USA 100:330-335.

Davies SW, Turmaine M, Cozens BA, DiFiglia M, Sharp AH, Ross CA, Scherzoner E, Wanker EE, Manginarini L, Bates GP (1997) Formation of neuronal intranulear inclusions underlies neurological dysfunction in mice transgenic for the mutation. Cell 90:537-548.

DiFiglia M, Sapp E, Chase K, Schwarz C, Meloni A, Young C, Martin E, Vonsattel J-P, Carraway R, Reeves SA, Boyce FM, Aronin N (1995) Huntingtin is a cytoplasmic protein associated with vesicles in human and rat brain neurons. Neuron 14:1075-1081.

DiFiglia M, Sapp E, Chase KO, Davies SW, Bates GP, Vonsattel JP, Aronin N (1997) Aggregation of huntingtin in neuronal intranuclear inclusions and dystrophic neuritis in brain. Science 277:1990-1993.

Engqvist-Goldstein AE, Kessels MM, Chopra VS, Hayden MR, Drubin DG (1999) An actin-binding protein of the Sla2/Huntingtin interacting protein 1 family is a novel component of clathrin-coated pits and vesicles. J Cell Biol 147:1503-1518.

Faber PW, Barnes GT, Srinidhi J, Guesella JF, MacDonald ME (1998) Huntingtin interacts with a family of WW domain proteins. Hum Mol Genet 7:1463-1474

Fernandez-Funez P, Nino-Rosales ML, de Gouyon B, She WC, Luchak JM, Martinez P, Turiegano E, Benito J, Capovilla M, Skinner PJ, McCall A, Canal I, Orr HT, Zoghbi HY, Botas J (2000) Identification of genes that modify ataxin-1-induced neurodegeneration. Nature 408:101-106.

Gafni J, Ellerby LM (2002) Calpain activation in Huntington's disease. J Neurosci 22:4842-4849.

Gervais FG, Singaraja RX, Anthoudakis S, Gutekunst C-A, Leavitt BR, Metzler M, Hackam AS, Tam J, Vaillancourt JP, Houtzager V, Rasper DM, Roy S, Hayden MR, Nicholson DW (2002) Recruitment and activation of caspase 8 by the huntingtin-interacting protein Hip-1 and a novel partner Hippi. Nat Cell Biol 4:95-105.

Gutekunst CA, Li SH, Yi H, Mulroy JS, Kuemmerle S, Jones R, Rye D, Ferrante RJ, Hersch SM, Li XJ (1999) Nuclear and neuropil aggregates in Huntington's disease: relationship to neuropathology. J Neurosci 19:2522-2534.

Hattula K, Peränen J (2000) FIP-2, a coiled-coil protein, links huntingtin to Rab8 and modulates cellular morphogenesis. Curr Biol 10:1603-1606.

Hazeki N, Tsukamoto T, Yazawa I, Koyama M, Hattori S, Someki I, Iwatsubo T, Nakamura K, Goto J, Kanazawa I (2002) Ultrastructure of nuclear aggregates formed by expressing an expanded polyglutamine. Biochem Biophys Res Commun 294:429-440.

Katzmann DJ, Odorizzi G, Emr SC (2002) Receptor downregulation and multivesicular-body sorting. Nat Rev Mol Cell Biol 3:893-905.

Kegel KB, Kim M, Sapp E, McIntyre C, Castaño JG, Aronin N, DiFiglia M (2000) Huntingtin expression stimulates endosomal-lysosomal activity, endosome tubulation, and autophagy. J Neurosci 20:7268-7278.

Khoshnan A, Ko J, Patterson PH (2002) Effects of intracellular expression of anti-huntingtin antibodies of various specificities on mutant huntingtin aggregation and toxicity. Proc Natl Acad Sci USA 99:1002-1007.

Kim M, Lee S-H, LaForet G, McIntyre C, Martin EL, Chang P, Kim TW, William M, Reddy PH, Tagle D, Boyce FM, Won L, Heller A, Aronin N, DiFiglia M (1999) Mutant huntingtin expression in clonal striatal cells: dissociation of inclusion formation and neuronal survival by caspase inhibition. J Neurosci 19:964-973.

Kim YJ, Yi Y, Sapp E, Wang Y, Cuiffo B, Kegel KB, Qin Z-H, Aronin N (2001) Caspase 3-cleaved N-terminal fragments of wild-type and mutant huntingtin are present in normal and Huntington's disease brains, associate with membranes, and undergo calpain-dependent proteolysis. Proc Natl Acad Sci USA 98:12784-12789.

Klionsky DJ (1999) Vacuolar import of proteins and organelles from the cytoplasm. Annu Rev Cell Biol 15:1-32.

Kuemmerle S, Gutekunst CA, Klein AM, Li XJ, Li SH, Beal MF, Hersch SM, Ferrante RJ (1999) Huntingtin aggregates may not predict neuronal death in Huntington's disease. Ann Neurol 46:842-849.

Kunz J, Schneider U, Howald I, Schmidt A, Hall MN (2000) HEAT repeats mediate plasma membrane localization of Tor $2 p$ in yeast. J Biol Chem 275:37011-37020.

Li H, Li S-H, Yu Z-X, Shelbourne P, Li XJ (2001) Huntingtin aggregateassociated axonal degeneration is an early pathological event in Huntington's disease mice. J Neurosci 21:8473-8481.

Liang XH, Jackson S, Seaman M, Brown K, Kempkes B, Hibshoosh H, Levine (1999) Induction of autophagy and inhibition of tumorigenesis by beclin 1. Nature 402:672-676.

Liu YF, Deth RC, Devys D (1997) SH3 domain-dependent association of huntingtin with epidermal growth factor receptor signaling complex. J Biol Chem 272:8121-8124.

Metzler M, Legendre-Guillemin V, Gan L, Chopra V, Kwok A, McPherson PS, Hayden MR (2001) HIP1 functions in clathrin-mediated endocytosis through binding to clathrin and adaptor protein 2. J Biol Chem 276:39271-39276.

Miake H, Mizusawa H, Iwatsubo T, Hasegawa M (2002) Biochemical characterization of the core structure of $\alpha$-synuclein filaments. J Biol Chem 277:19213-19219.

Modregger J, DiProspero NA, Charles V, Tagle DA, Plomann M (2002) Pac$\sin 1$ interacts with huntingtin and is absent from synaptic varicosities in presymptomatic Huntington's disease brains. Hum Mol Genet 11:2547-2558.

Panov AV, Gutekunst CA, Leavitt BR, Haden MR, Burke JR, Strittmatter WJ, Greenamyre JT (2002) Early mitochondrial calcium defects in Huntington's disease are a direct effect of polyglutamines. Nat Neurosci 5:731-736.

Passani LA, Bedford MT, Faber PW, McGinnis KM, Sharp AH, Gusella JF, Vonsattel JP, McDonald ME (2000) Huntingtin's WW domain partners in Huntingtin's disease post-mortem brain fulfill genetic criteria for direct involvement in Huntington's disease pathogenesis. Hum Mol Genet 9:2175-2182.

Perutz MF, Finch JT, Lesk A (2002) Amyloid fibers are water-filled nanotubes. Proc Natl Acad Sci USA 99:5591-5595.

Pitts KR, Yoon Y, Krueger EW, McNiven MA (1999) The dynamin-like protein DLP1 is essential for normal distribution and morphology of the endoplasmic reticulum and mitochondria in mammalian cells. Mol Biol Cell 10:4403-4417.

Poirier MA, Li H, Macosko J, Cai S, Amzel M (2002) Huntingtin spheroids and protofibrils as precursors in polyglutamine fibrilization. J Biol Chem 277:41032-41037.

Qin Z-H, Wang Y, Kikly KK, Sapp E, Kegel KB, Aronin N, DiFiglia M (2001) Pro-caspase- 8 is predominantly localized in mitochondria and released into cytoplasm upon apoptotic stimulation. J Biol Chem 276:8079-8086.

Qin Z-H, Wang Y, Kegel KB, Kazantsev A, Apostol BL, Thompson LM, Yoder J, Aronin H, DiFiglia M (2003) Autophagy regulates the processing of amino terminal huntingtin fragments. Hum Mol Gen 12:3231-3244.

Sapp E, Ge P, Aizawa H, Bird E, Penney J, Young AB, Vonsattel JP, DiFiglia M (1995) Evidence for a preferential loss of enkephalin immnoreactivity in the external globus pallidus in low grade Huntington's disease using high resolution image analysis. Neuroscience 64:397-404.

Sapp E, Schwarz C, Chase K, Bhide PG, Young AB, Penney J, Vonsattel JP, Aronin N, DiFiglia M (1997) Huntingtin localization in brains of normal and Huntington's disease patients. Ann Neurol 42:604-612.

Sapp E, Penney J, Young A, Aronin N, Vonsattel JP, DiFiglia M (1999) Axonal transport of N-terminal huntingtin suggests early pathology of corticostriatal projections in Huntington disease. J Neuropathol Exp Neurol 58:165-173.

Saudou F, Finkbeiner S, Devys D, Greenberg ME (1998) Huntingtin acts in the nucleus to induce apoptosis but death does not correlate with the formation of intranuclear inclusions. Cell 95:55-66.

Scherzinger E, Lurz R, Turmaine M, Mangiarini L, Hollenbach B, Hasenbank R, Bates GP, Davies SW, Lhrach H, Wanker EE (1997) Huntingtinencoded polyglutamine expansions form amyloid-like protein aggregates in vitro and in vivo. Cell 90:549-558. 
Sever S (2002) Dynamin and endocytosis. Curr Opin Cell Biol 14:463-467. Sisodia SS (1998) Nuclear inclusions in glutamine repeat disorders: are they pernicious, coincidental, or beneficial? Cell 95:1-4.

Sittler A, Walter S, Wedemeyer N, Hasenbank R, Scherzinger E, Eickhoff H, Bates GP, Lehrach H, Wanker EE (1998) SH3GL3 associates with the huntingtin exon 1 protein and promotes the formation of polyglncontaining protein aggregates. Mol Cell 2:427-436.

So CW, Sham MH, Chew SL, Cheung N, So CKC, Chung SK, Caldas C, Wiedemann LM, Chan LC (2000) Expression and protein-binding studies of the EEN gene family, new interacting partners for dynamin, synaptojanin and huntingtin proteins. Biochem J 348:447-458.

Takano H, Gusella JF (2002) The predominantly HEAT-like motif structure of huntingtin and its association and coincident nuclear entry with dorsal, an NF- $\kappa \mathrm{B} / \mathrm{Rel} /$ dorsal family transcription factor. BMC Neurosci 3:15.

Velier J, Kim M, Schwarz C, Kim TW, Sapp E, Chase K, Aronin N, DiFiglia M (1998) Wild-type and mutant huntingtins function in vesicle trafficking in the secretory and endocytic pathways. Exp Neurol 152:34-40.

Vonsattel JP, DiFiglia M (1998) Huntington disease. J Neuropathol Exp Neurol 57:369-384.

Vonsattel JP, Myers RH, Stevens TJ, Ferrante RJ, Bird ED, Richardson Jr EP (1985) Neuropathological classification of Huntington's disease. J Neuropathol Exp Neurol 44:559-577.
Waelter S, Scherzinger E, Hasenbank R, Nordhoff E, Lurz R, Goehler H, Gauss C, Sathasivam K, Bates GP, Lehrach H, Wanker EE (2001) The huntingtin interacting protein 1 is a clathrin and $\alpha$-adaptin-binding protein involved in receptor-mediated endocytosis. Hum Mol Genet 10:1807-1817.

Wainwright MS, Perry BD, Won LA, O'Malley KL, Wang WY, Ehrlich ME, Heller A (1995) Immortalized murine striatal neuronal cell lines expressing dopamine receptors and cholinergic properties. J Neurosci 15:676-688.

Walsh DM, Klyubin I, Fadeeva JV, Cullen WK, Anwyl R, Wolfe MS, Rowan MJ, Selkoe DJ (2002) Naturally secreted oligomers of amyloid $\beta$ protein potently inhibit hippocampal long-term potentiation in vivo. Nature 416:535-539.

Wanker E, Rovira C, Scherzinger E, Hasenbank R, Wälter S, Tait D, Colicelli J, Lehrach H (1997) HIP-1: a huntingtin interacting protein isolated by the yeast two-hybrid system. Hum Mol Genet 6:487-495.

Wellington CL, Ellerby LM, Rogers CAD, Warby S, Graham RK, Loubser O, van Raamsdonk J, Singaraja R, Yang YZ, Gafni J, Bredesen D, Hersch SM, Leavitt BR, Roy S, Nicholson DW, Hayden MR (2002) Caspase cleavage of mutant huntingtin precedes neurodegeneration in Huntington's disease. J Neurosci 22:7862-7872. 